\title{
Chapter 1 \\ Operators, Algebras and their Invariants for Aperiodic Tilings
}

\author{
Johannes Kellendonk
}

\begin{abstract}
We review the construction of operators and algebras from tilings of Euclidean space. This is mainly motivated by physical questions, in particular after topological properties of materials. We explain how the physical notion of locality of interaction is related to the mathematical notion of pattern equivariance for tilings and how this leads naturally to the definition of tiling algebras. We give a brief introduction to the $K$-theory of tiling algebras and explain how the algebraic topology of $K$-theory gives rise to a correspondence between the topological invariants of the bulk and its boundary of a material.
\end{abstract}

\subsection{Tilings and the topology of their hulls}

In condensed matter theory tilings are used to describe the spatial arrangement of the constitutents which make up a material, for instance a quasicrystal. They describe the spatial structure of the material.

Associated to a tiling are various topological spaces and topological dynamical systems. Their topology is peculiar. It takes into account the topology of the space in which the tiling lies and, at the same time, its pattern structure, that is, the way how finite patterns repeat over the tiling. Continuity in the tiling topology is related to locality in physics.

Johannes Kellendonk

Institute Camille Jordan, Université Claude Bernard Lyon1, F-69622 Villeurbanne, e-mail: kellendonk@math.univ-lyonl.fr 


\subsubsection{Basic notions}

There exist various introductions to the theory of aperiodic tilings which cover the basic notions we need it, for instance $[40,4]$. We recall here only the strict necessary.

- A tiling is a covering of $\mathbb{R}^{d}$ by tiles whose interiors do not overlap.

- A tile is here a possibly decorated compact convex polyhedron. The polyhedron (up to translational congruence) is its shape. The decoration is by symbols from a symbol set $\mathcal{A}$ and serves to distinguish equal shapes (or enforce matching conditions). We assume that tiles match face to face.

- The tiles of a tiling at different positions are considered different. If the tiling has only finitely many tiles up to translation and only finitely many symbols then it is said to have finite local complexity (FLC).

Examples are Voronoi tilings (or their dual) coming from Delone sets.

A tiling $\mathcal{T}$ can be translated by a vector $x \in \mathbb{R}^{d}$ in the space it lies. By this we mean that the individual tiles are shifted by $x$. We denote the tiling translated by $x$ as $\mathcal{T}+x$ (the tile which has been on the origin 0 of the space in which $\mathcal{T}$ lies is now on $x$ ). A period of a tiling is a vector $x \in \mathbb{R}^{d}$ such that $\mathcal{T}+x=\mathcal{T}$. Aperiodicity may occur through the shapes of the tiles, like for instance in the Penrose tilings, or through the decorations, or through both. In order not to overburden the notation and complexity we now consider the first two cases separately and only indicate how to do the third.

\subsubsection{Undecorated tilings}

An undecorated tiling by compact convex polyhedra without decorations can be equivalently described as the closed subset of $\mathbb{R}^{d}$ given by the union of the boundaries of its tiles. In the following we attach a language and a hull to any closed subset of $\mathbb{R}^{d}$.

Let $\mathcal{T}$ be a closed subset of $\mathbb{R}^{d}$. For $R \geq 0$ and $x \in \mathbb{R}^{d}$, the $R$-patch of $\mathcal{T}$ centered at $x$ is intersection of the $R$-ball at $x$ with $\mathcal{T}$, union with the boundary of the $R$-ball,

$$
B_{R}[\mathcal{T} ; x]:=\left(B_{R}(x) \cap \mathcal{T}\right) \cup \partial B_{R}(x) .
$$

The $R$-patch class of an $R$-patch $B_{R}[\mathcal{T} ; x]$ is its translational congruence class. The representative of the $R$-patch class of $B_{R}[\mathcal{T} ; x]$ at 0 is $B_{R}[\mathcal{T} ; x]-x=$ $B_{R}[\mathcal{T}-x ; 0]$, we simply denote it by $B_{R}[\mathcal{T}-x]$.

Using $R$-patches we define a metric-topology on the set of all closed subsets of $\mathbb{R}^{d}$. Let $\mathcal{T}, \mathcal{T}^{\prime}$ be two closed subsets, their distance is

$$
D\left(\mathcal{T}, \mathcal{T}^{\prime}\right)=\inf \left\{\epsilon: d_{H}\left(B_{\epsilon^{-1}}[\mathcal{T}-x], B_{\epsilon^{-1}}\left[\mathcal{T}^{\prime}-x\right]\right) \leq \epsilon\right\}
$$


where $d_{H}$ is the Hausdorff distance between the set of all compact subsets of $B_{\epsilon^{-1}}(0)$ which contain $\partial B_{\epsilon^{-1}}(0)$. We call $\mathcal{L}(\mathcal{T})=\bigcup_{R \geq 0} \mathcal{L}^{R}(\mathcal{T})$,

$$
\mathcal{L}^{R}(\mathcal{T})=\overline{\left\{B_{R}[\mathcal{T}-x]: x \in \mathbb{R}^{d}\right\}}{ }^{d}
$$

the language of $\mathcal{T}$. We say that a closed subset $\mathcal{T}^{\prime}$ of $\mathbb{R}^{d}$ is allowed for the language of $\mathcal{T}$ if each $R$-patch-class of $\mathcal{T}^{\prime}$ occurs in $\mathcal{L}(\mathcal{T})$. The set of all closed subsets which are allowed for $\mathcal{T}$ is called the continuous hull of $\mathcal{T}$ and denoted by $\Omega(\mathcal{T})$. There are maps $\mathcal{L}^{R}(\mathcal{T}) \ni B_{R}\left[\mathcal{T}^{\prime}\right] \mapsto B_{R^{\prime}}\left[\mathcal{T}^{\prime}\right] \in \mathcal{L}^{R^{\prime}}(\mathcal{T})$, for $R^{\prime} \leq R$ and $\Omega(\mathcal{T})$ can be seen as the inverse limit of these maps. In particular, the metric topology on $\Omega(\mathcal{T})$ is the same as the inverse limit topology, namely it is the smallest topology so that all the maps $\Omega(\mathcal{T}) \ni \mathcal{T}^{\prime} \mapsto B_{R}\left[\mathcal{T}^{\prime}\right] \in \mathcal{L}^{R}(\mathcal{T})$ are continuous.

Examples:

1. If $\mathcal{T}$ is bounded then $\Omega(\mathcal{T})$ is the $d$-sphere.

2. If $\Lambda$ is a regular lattice in $\mathbb{R}^{d}$ and $\mathcal{T}$ has period lattice $\Lambda$ then $\Omega(\mathcal{T})$ is the torus $\mathbb{R}^{d} / \Lambda$.

3. If $\mathcal{T}$ is a hyperplane of dimension $k$ then $\Omega(\mathcal{T})=\mathbb{R}^{k} \times S^{d-k}$.

Applied to an undecorated tiling this construction yields its continuous hull. If $\mathcal{T}$ has FLC then $\left\{B_{R}[\mathcal{T}-x]: x \in \mathbb{R}^{d}\right\}$ is already closed in the Hausdorff topology and so any $R$-patch of $\mathcal{L}^{R}(\mathcal{T})$ has a translate occuring in $\mathcal{T}$. Then $\Omega(\mathcal{T})$ is the set of all tilings of $\mathbb{R}^{d}$ whose $R$-patches occur somewhere in $\mathcal{T}$.

\subsubsection{Wang tilings}

Consider an (undecorated) tiling $\mathcal{T}$ whose vertex set $\mathcal{V}$ happens to lie in a regular lattice $\Lambda \subset \mathbb{R}^{d}$. Choose a closed fundamental domain $I$ (a parallel epiped) for $\Lambda$ and superimpose $\mathcal{T}$ with the periodic tiling whose tiles are $I+\lambda, \lambda \in \Lambda$. Then the boundary points of the tiles of $\mathcal{T}$ mark on the tiles of the periodic tiling patterns which we can take as symbols, and thus our tiling $\mathcal{T}$ can be alternatively described by a tiling by decorated parallel epipeds. The two tilings contain the same information, they can be transformed into each other by inspection of their $R$-patches. This is an example of mutual local derivability [5] the definition of which we recall further down. The tiling by decorated parallel epipeds is a so-called Wang tiling.

A Wang tiling is a tiling in which all tiles are cubes meeting face to face (or a parallel epipeds, but we can always apply a linear transformation to deform it into a cube), but the tiles carry decorations ${ }^{1}$ and so Wang tilings may be aperiodic. Once we have fixed which tile contains the origin 0 of our

1 Originally the decorations encode matching conditions, but we will not make use of that here. 
space, the set of tiles of the tiling are in bijective correspondence to $\mathbb{Z}^{d}$ and their decorations may be described by a map into a space of decorations, or symbols, $\xi: \mathbb{Z}^{d} \rightarrow \mathcal{A}$. This suggests a more symbolic approach to the construction of the hull, and so we start with the description of the symbolic hull of a Wang tiling. We suppose that $\mathcal{A}$ is a compact set and if it is finite the Wang tiling is said to have finite local complexity. The (symbolic) $N$-patch of a Wang tiling at $n \in \mathbb{Z}^{d}$ is the decoration of the cube of size $2 N+1$ at $n$, more precisely, this is the subset $\left\{\xi_{n+m}:\|m\|_{\max } \leq N\right\} \subset \mathcal{A}^{[-N, N]^{d}}$. The (symbolic) language of $\xi$ is $\ell(\xi)=\bigcup_{N} \ell^{N}(\xi)$ where $\ell^{N}(\xi)$ is the closure of the set of $N$-patches of $\xi$ (shifted to 0 ) in $\mathcal{A}^{[-N, N]^{d}}$. We can make the same inverse limit construction as above to obtain the symbolic hull of the Wang tiling which we denote $\Xi(\xi)$ or simply $\Xi$ if the context is clear.

The additional information which is needed to construct from a the map $\xi: \mathbb{Z}^{d} \rightarrow \mathcal{A}$ a tiling of $\mathbb{R}^{d}$ is the location of the origin, that is, a choice of point $x$ in the cube $I$. Given such a point the Wang tiling symbolized by $\xi$ is placed in $\mathbb{R}^{d}$ in such a way that the point $x$ lies on the origin of our space if we identify $I$ with the tile corresponding to $0 \in \mathbb{Z}^{d}$ (so with symbol $\xi_{0}$ ). The continuous hull $\Omega$ of the Wang tiling is then the quotient of $I \times \Xi$ by the following relation: If $x$ belongs to the boundary of $I$ then the origin of our space will be in two or more tiles of the Wang tiling. These possibilities have to be identified. More precisely, if $\alpha: \mathcal{A}^{\mathbb{Z}^{d}} \rightarrow \mathcal{A}^{\mathbb{Z}^{d}}$ denotes the translation action, $\alpha_{m}(\xi)(n)=\xi(n-m)$, then

$$
\Omega=\mathbb{R}^{d} \times_{\mathbb{Z}^{d}} \Xi
$$

which is the quotient of the cartesian product w.r.t. the diagonal action, i.e. w.r.t. the relation $(x, \xi) \sim\left(x+m, \alpha_{m}(\xi)\right)$. The continuous hull $\Omega$ is thus a fibre bundle over the $d$-torus $\mathbb{R}^{d} / \mathbb{Z}^{d}$ with typical fibre $\Xi$.

\subsubsection{Pattern equivariant functions and local derivability}

The notion of pattern equivariant functions has been introduced for tilings of finite local complexity in [26]. We extend here this notion to arbitrary tilings.

Let $\mathcal{T}$ be a closed subset. A map $f$ from $\mathbb{R}^{d}$ to a topological space $Y$ is called strongly pattern equivariant for $\mathcal{T}$ (or local) if there exist $R \geq 0$ and a continuous function $b: \mathcal{L}^{R}(\mathcal{T}) \rightarrow Y$ (a sort of continuous sliding block code) such that

$$
f(x)=b\left(B_{R}[\mathcal{T}-x]\right) .
$$

In particular, if the $R$-patch of $\mathcal{T}$ at $x$, when shifted by the vector $y-x$ coincides with the $R$-patch of $\mathcal{T}$ at $y$ then $f(x)=f(y)$. If $Y$ is a metric space 
then we define (weakly) pattern equivariant functions as functions from $\mathbb{R}^{d}$ to $Y$ which are uniform limits of strongly pattern equivariant functions.

A closed subset $\mathcal{T}^{\prime}$ is locally derivable from a closed subset $\mathcal{T}$ if there exists $R>0$ and a continuous function $b: \mathcal{L}^{R}(\mathcal{T}) \rightarrow \mathcal{L}^{1}\left(\mathcal{T}^{\prime}\right)$ (a local rule) such that

$$
B_{1}\left[\mathcal{T}^{\prime}-x\right]=b\left(B_{R}[\mathcal{T}-x]\right)
$$

for all $x \in \mathbb{R}^{d}$. In other words, we can construct the 1-patch of $\mathcal{T}^{\prime}$ at $x$ from the $R$-patch class of $\mathcal{T}$ at $x$. If $\mathcal{T}^{\prime}$ is locally derivable from $\mathcal{T}$ and vice versa we say that the two are mutually locally derivable. Since (1.1) has to hold for all $x \in \mathbb{R}^{d}$ there is, for each $r>0$, a unique extension $b_{r}: \mathcal{L}^{R+r}(\mathcal{T}) \rightarrow \mathcal{L}^{1+r}\left(\mathcal{T}^{\prime}\right)$ such that $B_{1+r}\left[\mathcal{T}^{\prime}-x\right]=b_{r}\left(B_{R+r}[\mathcal{T}-x]\right)$. The $b_{r}$ are also continuous and so a local derivation extends to a continuous map from the hull of $\mathcal{T}$ to the hull of $\mathcal{T}^{\prime}$.

Note that if $S$ is locally derivable from $\mathcal{T}$, then the local rule $b$ allows to locally derive a set $S\left(\mathcal{T}^{\prime}\right)$ for any other element of $\Omega(\mathcal{T})$. Any locally derivable uniformly discrete subset $S$ from $\mathcal{T}$ defines a transversal

$$
\Xi_{S}=\left\{\mathcal{T}^{\prime} \in \Omega: 0 \in S\left(\mathcal{T}^{\prime}\right)\right\}
$$

Since $s \in S\left(\mathcal{T}^{\prime}\right)$ if and only if $0 \in S\left(\mathcal{T}^{\prime}-s\right)$ we see that

$$
S\left(\mathcal{T}^{\prime}\right)=\left\{x \in \mathbb{R}^{d}: \mathcal{T}^{\prime}-x \in \Xi_{S}\right\}
$$

Clearly, the vertex set $\mathcal{V}$ of a tiling $\mathcal{T}$ is locally derivable. We call $\Xi_{\mathcal{V}}$ the canonical transversal of $\Omega(\mathcal{T})$.

Let $F: \mathbb{R}^{d} \rightarrow \mathbb{R}^{d}$ be a differentiable function whose differential $d F$ is strongly pattern equivariant for an undecorated tiling $\mathcal{T}$. We can apply $F$ to the vertex set $\mathcal{V}$ of $\mathcal{T}$ to obtain a new set $\mathcal{V}^{\prime}=F(\mathcal{V})$ which we would like to interprete as vertex set of a new tiling $\mathcal{T}^{\prime}$. If $d F$ is close enough to the identity we can do this by (a) requiring that the new tiles are the convex hulls of their vertices, and (b) preserving the combinatorial structure, that is, the information which vertices are vertices of the same tile. One may think of $F$ as being homotopic to the identity and the new tiling to be obtained by a continuous deformation. At least if $\mathcal{T}$ is FLC it can be shown that, if $d F$ is close enough to the identity then $F$ is invertible and $d F^{-1}$ is strongly pattern equivariant for $\mathcal{T}^{\prime}$. This then implies that $F$ induces a homeomorphism $\mathcal{F}: \Omega(\mathcal{T}) \rightarrow \Omega\left(\mathcal{T}^{\prime}\right)$ which maps $\Xi_{\mathcal{V}}(\mathcal{T})$ to $\Xi_{\mathcal{V}^{\prime}}\left(\mathcal{T}^{\prime}\right)$. Using this technique of deformation [10,27] one can show the following result.

Theorem 1 ([42]). Let $\mathcal{T}$ be a tiling of finite local complexity. There exists a tiling $\mathcal{T}^{\prime}$ of finite local complexity whose vertices lie in a regular lattice and a homeomorphism between the continuous hulls of $\mathcal{T}$ and $\mathcal{T}^{\prime}$ which preserves the canonical transversals.

Up to homeomorphisms preserving the canonical transversals any tiling of FLC can therefore be understood as a Wang tiling. Indeed, if the vertices $\mathcal{V}^{\prime}$ 
lie in a regular lattice $\Lambda$ then $\Lambda$ must be locally derivable from $\mathcal{T}^{\prime}$ and so we may identify $\Xi_{\Lambda}\left(\mathcal{T}^{\prime}\right)$ with $\{0\} \times \Xi$ where $\Xi$ is the symbolic hull of the Wang tiling described above. It then follows that the continuous hulls of $\mathcal{T}^{\prime}$ and of the Wang tiling also agree.

\subsection{Operators from tilings}

We are interested in wave phenomena in a media whose structures are modeled by tilings. The waves might either be probability waves of quantum mechanics, or classical waves, like light or acoustic waves. The waves are solutions of wave equations, like the Schrödinger equation or the Helmholtz equation, which typically are differential equations. Assuming that the waves satisfy the superposition principle and the equations are linear, the stationary problem consists of solving an eigenvalue equation in the Hilbert space $L^{2}\left(\mathbb{R}^{d}\right)$, or more precisely to determine the spectrum of a linear differential operator $H$ on $L^{2}\left(\mathbb{R}^{d}\right)$.

If no external forces are present then the locality principle requires that the differential operator $H$ has the form

$$
H=\sum_{\alpha} f_{\alpha} D^{\alpha}
$$

where $D^{\alpha} \psi=\prod_{i=1}^{d}\left(\frac{\partial}{\partial x_{i}}\right)^{\alpha_{i}} \psi$ for $\alpha \in \mathbb{N}^{d}$, and the coefficients $f_{\alpha}$ are pattern equivariant functions on $\mathbb{R}^{d}$ for the underlying tiling, typically non-zero only for a finite number of $\alpha$. External magnetic fields can be incorporated by minimal coupling, but we won't consider that case here.

$H$ is typically an unbounded operator on $L^{2}\left(\mathbb{R}^{d}\right)$ which requires a certain amount of care, in partcular as we will want to attach it to a $C^{*}$-algebra. This could be done by considering bounded functions of $H$, like the heatkernel $\exp (-t H)$ or the resolvent $(H-x)^{-1}$, but alternatively by considering so-called tight binding models whose operators are from the start bounded. This is what we will do here.

\subsubsection{Tight binding operators}

Let $S$ be a Delone set which is locally derivable from a tiling $\mathcal{T}$. For instance, the vertex set of $\mathcal{T}$ is locally derivable from $\mathcal{T}$. A tight binding operator is an operator on $\ell^{2}\left(S, \mathbb{C}^{N}\right)$ of the form

$$
H \psi(x)=\sum_{y \in S} H_{x y} \psi(y)
$$


where, for each pair $(x, y), H_{x y}$ is an $N \times N$ matrix. If there is an $R>0$ such that $H_{x y}=0$ provided $|y-x|>R$ the model has finite range and convergence of the sum is not an issue. The locality principle now translates into the requirement that the $H_{x y}$ are pattern equivariant in the following sense: We call double $R$-patch at $(x, y) \subset \mathbb{R}^{d} \times \mathbb{R}^{d}$ the set

$$
B_{R}[\mathcal{T} ; x, y]:=B_{R}[\mathcal{T} ; x] \times B_{R}[\mathcal{T} ; y] \subset \mathbb{R}^{d} \times \mathbb{R}^{d}
$$

and its class $\left[B_{R}[\mathcal{T} ; x, y]\right]$ the equivalence class under the diagonal action of $\mathbb{R}^{d},\left(t, t^{\prime}\right)+x=\left(t+x, t^{\prime}+x\right)$. The distance $\|x-y\|$ between $x$ and $y$ is called the range of the double $R$-patch at $(x, y)$. The set of double $R$-patch classes of closed subsets to $\mathbb{R}^{d}$ is a metric space w.r.t. the Hausdorff metric ${ }^{2}$. A function $\mathbb{R}^{d} \times \mathbb{R}^{d} \ni(x, y) \mapsto Y$ is then strongly pattern equivariant if, for some $R, M>0$, there is a continuous function $b$ defined on the set double $R$-patch classes with range smaller or equal to $M$ such that $f(x, y)=b\left(\left[B_{R}[\mathcal{T} ; x, y]\right]\right)$.

A simple example of an operator of the form (1.2) is the discrete Laplacian on the Penrose tiling. Here $S$ is the vertex set of the tiling, $N=1, H_{x y}=1$ if $x$ and $y$ are vertices of a common edge whereas $H_{x y}=0$ otherwise.

\subsubsection{Tight binding operators for Wang tilings}

If the vertex set of $\mathcal{T}$ lies in a regular lattice $\Lambda$ then we may take $S=\Lambda \cong \mathbb{Z}^{d}$ above and rewrite (1.2) as

$$
H \psi(x)=\sum_{n \in \mathbb{Z}^{d}} H_{n}(x) \psi(x-n)
$$

for $\psi \in \ell^{2}\left(\mathbb{Z}^{d}, \mathbb{C}^{N}\right)$, where $H_{n}(x)=H_{x x-n} . H$ has finite rank if $H_{n}=0$ once $n$ is larger than a given size. The above notion of pattern equivariance using double $R$-classes simplifies and we only have to make sure that $H_{n}: \mathbb{Z}^{d} \rightarrow$ $M_{N}(\mathbb{C})$ is strongly pattern equivariant for each $n \in \mathbb{Z}^{d} . H$ depends thus only on the symbolic sequence $\xi: \mathbb{Z}^{d} \rightarrow \mathcal{A}$ of the Wang tiling derived from $\mathcal{T}$ and $S$. Using the translation operators on $\mathbb{Z}^{d}, T^{n} \psi(x)=\psi(x-n)$ we can write

$$
H=\sum_{n \in \mathbb{Z}^{d}} H_{n} T^{n}
$$

By Theorem 1, any operator of the form (1.2) on a tiling of FLC can be brought into the form (1.3). This applies, for instance, also to the discrete Laplacian on the Penrose tiling, even though the version written in the form (1.3) might look a lot more complicated and does not display its fivefold

2 the Hausdorff metric between equivalence classes of compact sets is the infimum over the Hausdorff distances between representatives of the classes 
symmetry. But the form (1.3) has important structural consequences which, in particular, allow us to work with crossed product algebras instead of more general groupoid algebras.

\subsubsection{A simple class of one-dimensional models}

To illustrate the different phenomena which can occur in different type of tilings we consider four simple tight binding models defined on onedimensional Wang tilings. The reader may consult [29] for more details.

Given a symbolic sequence $\xi \in \mathcal{A}^{\mathbb{Z}}$ over some symbol space $\mathcal{A}$, we consider the following operators on $\ell^{2}\left(\mathbb{Z}, \mathbb{C}^{N}\right)$.

1. $H=H_{0}+T+T^{*}$ with $H_{0}(n)=b\left(\xi_{n}\right)$ for some real block sliding code $b: \mathcal{A} \rightarrow \mathbb{R}$ of range $0(N=1) . H$ is the discrete Laplacien on $\mathbb{Z}^{d}$ plus an onsite potential which depends only on the symbol at site $x$.

2. $H=\left(\begin{array}{cc}0 & Q^{*} \\ Q & 0\end{array}\right)$ with $Q=\sum_{n \in \mathbb{Z}} Q_{n} T^{n}$ with $Q_{n}(m)=b_{n}\left(\xi_{m}\right)$ for complex block sliding codes of finite range $b_{n}: \ell^{L}(\mathcal{A}) \rightarrow \mathbb{C}$, only finitely many being non-zero $(N=2)$. $H$ is the Hamlitonian of a typical one dimensional tight binding model of finite range with chiral symmetry, namely $H$ anti-commutes with the self adjoint unitary $\Gamma=\left(\begin{array}{cc}1 & 0 \\ 0 & -1\end{array}\right)$.

The sequences $\xi$ we consider here are obtained from a cut \& project scheme with dimension and codimension equal to one, namely they are constructed as follows. For a given irrational number $\theta \in[0,1]$ we consider the Kronecker flow line

$$
L:=\left\{\left(t, \theta^{\prime} t\right) \mid t \in \mathbb{R}\right\} / \mathbb{Z}^{2}
$$

on the torus $\mathbb{R}^{2} / \mathbb{Z}^{2}$ with slope $\theta^{\prime}=\frac{\theta}{1-\theta}$ (see the red sloped line in the pictures $(1-3))$. A window $W$ is a (not necessarily connected) curve in the torus which is transversal to the flow in the sense that there exists $\epsilon>0$ such that for every point $w \in W$ and all $0<|t|<\epsilon, w+\left(t, \theta^{\prime} t\right) \neq W$. We consider three types of windows. In picture (1) $W$ is a closed continuous (blue) curve with a single slope, in (2) $W$ a closed continuous curve with more than one slope. Finally in Picture (3) $W$ has a single slope but is not a closed loop. It makes a jump, and we suppose that the boundary points of $W$ belong to $W$ and lie on a line parallel to $L$, but not equal to $L$. 


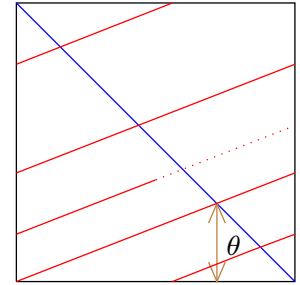

(1)

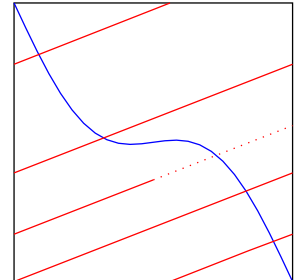

(2)

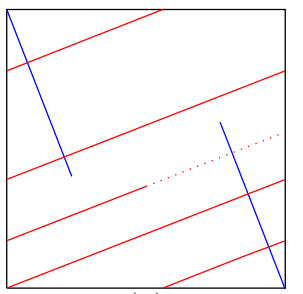

(3)

We parametrize the intersection of $L$ with window $W$ by a sequence of real numbers $\left(t_{n}\right)_{n \in \mathbb{Z}}$, that is, $\left(t_{n}, \theta^{\prime} t_{n}\right) \in W+\mathbb{Z}^{2}$, ordered according to the standard order of the real line (there is an irrelevant choice of which element is the zeroth one). The symbolic sequence we are looking at is $\xi_{n}=t_{n+1}-t_{n}$, so $\mathcal{A}$ is a compact subset of $\mathbb{R}^{+}$.

If one computes the symbolic hull $\Xi$ of $\xi$ one finds the following. Except for an important subtlety in the third case, all elements of the hull can be obtained by moving $W$ relative to $L$ and repeating the same construction: take $s \in S:=\{(t, 1-t) \mid 0 \leq t<1\}$, parametrize the intersection of $L$ with $s+W,\left(t_{n}, \theta^{\prime} t_{n}\right) \in s+W+\mathbb{Z}^{2}$ and set $\xi_{n}(s)=t_{n+1}-t_{n}$. The choice of zeroth element coherent by demanding it to change continuously with $s$, as long as $s$.

1) If the window $W$ is a closed continuous curve with a single slope then the sequence $\xi$ is periodic and the hull consists of a single point. The models introduced above with periodic $\xi$ have a band spectrum.

2) If the transversal $W$ is a closed continuous curve with more than one slope, then the sequence $\xi$ is aperiodic and takes infinitely many values (it has infinite local complexity). Sequences of this type are almost periodic in the sense of Bohr. Physically they describe incommensurate structures. With an appropriate shape for $W$ and $b\left(\xi_{n}\right)=\lambda \xi_{n}$ the operator $H=H_{0}+T+T^{*}$ is the Harper model. The computation of its spectral properties has a long history [33]. Its spectrum is a Cantor set [3]. Its spectral type depends on the value of $\lambda$. Below a critical value for $\lambda$ the operator has absolute continuous spectrum, at the critical value it has singular continuous spectrumn, and above that critical value it has pure point spectrum (for almost all $\theta$ ) [23].

The map $S \ni s \mapsto \xi(s) \in \Xi$ is bijective and continuous and so yields a homeomorphism between the circle $S$ and the symbolic hull $\Xi$. We may parametrize $S$ in such a way that the shift action on $\Xi$ corresponds to the rotation by $\theta$.

3) If the window $W$ is a discontinuous curve with a single slope then the sequences $\xi$ are so-called Sturmian sequences. They are aperiodic and have finite local complexity. They are also referred to as quasi-periodic, because such structures are used for one dimensional quasicrystals. With the choice of $W$ as in the picture (3) there are only two possible values for $\xi_{n}$, let's call them $a$ and $b, a>b$. If the onsite potential of the above model $H=$ 
$H_{0}+T+T^{*}$ takes different values on $a$ and $b$, we have the so-called Kohmoto model. Its spectrum is expected to be a Cantor set and singular continuous; this could be rigorously proven for the value of $\theta$ which corresponds to the Fibonacci sequence [14].

The jumps in $W$ as in Picture (3) lead to an important subtlety. If the line $L$ passes through one boundary point of $s+W$ then it passes also through the other boundary point. This situation will occur whenever $L \cap(s+\partial W) \neq \emptyset$, which happens for a countable dense set of values for $s \in S$. For these values, which we call singular, $\xi(s)$ will contain a third length $c$ and therefore not in the symbolic hull of the original sequence $\xi$. However, by selecting only one of the two boundary points one obtains an element of the hull. Stated in terms of sequences, if $s$ is singular then $\xi(s)$ contains somewhere the word $b c b$. If we replace this word by either $a b$ or $b a$ then the resulting sequence will be in the symbolic hull $\Xi$. As a consequence, $\Xi$ contains the sequences $\xi(s)$ coming from the points of $S$ which are not singular and, for each singular point of $S$, two sequences corresponding to the above two choices. The sequences of $\Xi$ which come from singular points of $S$ can be characterised by the fact that they contain a pair $a b$ (or $b a$ ) which can be flipped to $b a$ (or $a b$, respectively) so that the result lies still in $\Xi$. Such a flip is called a phason flip. It plays an important role in quasi-crystal physics.

Topologically, $\Xi$ is the circle $S$ disconnected along its singular points [17]. By disconnecting an interval $[0,1]$ at some point $0<r<1$ we mean the following: We take $r$ out to get two half open intervals and then add to each half open interval individually its missing boundary point. The result is the disjoint union of $[0, r]$ with $[r, 1]$ which hence has two connected components. There is an obvious map from this disjoint union back to the interval, just identify the two added boundary points.

Using inverse limits one can perform this procedure to a circle for a countable subset points. If this subset is dense then the resulting disconnected circle, which we denote $S_{c}$, is totally disconnected, the connected component of a point will contain only that point. There will then be a map from the disconnected circle $S_{c}$ to the original circle $S$ which is almost everywhere one to one, and two to one at the singular points.

The shift action on the hull can be described on $S_{c}$ as follows: on the non-singular points of $S$ the action is by rotation by $\theta$, as in the almost periodic case, and the single orbit of singular points of $S$ corresponds to two orbits in $S_{c}$.

4) In the context of the above situation there is another possibilty to resolve the issue of a third length $c$ appearing in $\xi(s)$ for singular $s$ [28]. We explain this the idea, which goes back to [16], first for the interval $[0,1]$ which we disconnected above at some interior point $r$. Instead of adjoing two boundary points to the half-open intervals $[0, r)$ and $(r, 1]$ to make them closed and disconnected we may add an intervall $[0,1]$ whose boundary points become the missing boundary points of the half-open intervals. The 
result is then a single (connected) interval $[0, r) \cup[r, r+1] \cup(r+1,2]=[0,2]$. When doing this for the circle with infinitely many points the result will be a again a circle, which we call the augmented circle $S_{a u g}$. It seems that the augmented circle has infinite circumference, but since we are only interested in its topology this is irrelevant. For the symbolic sequences $\xi$ this has the following interpretation. If $\xi(s)$ is singular and thus contains the forbidden word $b c b$ then, apart from the choices $a b$ and $b a$, we have the possibility to replace $b c b$ with $a_{t} b_{t}$ where $a_{t}=(1-t) a+t b$ and $b_{t}=$ $t a+(1-t) b, t \in[0,1]$. This enlarges the symbol set to $\mathcal{A}=[a, b]$. For $t \neq 0,1$ the resulting sequences are no longer Sturmian, but the augmentation has a nice physical interpretation; it corresponds to making the phason flips a continuous motion.

This has consequences for the hull with its shift action. The augmented hull $\tilde{\Xi}$ is by definition the set of sequences obtained in the above way, that is, it contains $\Xi$ together with a countable set of intervals. Topologically we may identify it with $S_{a u g}$ and then the shift action corresponds to, the rotation by $\theta$ on the points which are not singular, and a permutation of the added in intervals. Indeed, if we require that the $t$-variable is kept fixed there is only one possible continuous extension of the $\mathbb{Z}$-action to $S_{\text {aug }}$. The effect on the spectrum of the associated Hamiltonian has been numerically analysed in [28]. If the underlying sequence contains $a_{t} b_{t}$ for $t \neq 0,1$, the spectrum contains an additional eigenvalue in each gap.

\subsubsection{Gaps and the integrated density of states}

A gap in the spectrum of self adjoint operators is a connected component of its complement in $\mathbb{R}$. Physically a gap means that the material cannot have (electronic or wave) states at energies which lie in the gap ${ }^{3}$. In other words, the density of states vanishes in the gaps of the spectrum. The integrated density of states at energy $E, \operatorname{IDS}(E)$, is the integral over the density of states from $-\infty$ to $E$. It is thus a positive increasing function which is constant on gaps. Under suitable homogeneity assumptions on the material (for instance, if the tiling dynamical system is uniquely ergodic) the integrated density of states at $E$ can be expressed as the trace per unit volume of $P_{\leq E}(H)$, the spectral projection of $H$ to its states with energy lower than $E$. The trace per unit volume is a linear functional which can be evaluated on operators of $\ell^{2}\left(\mathbb{Z}^{d}\right)$ (provided they are trace-class w.r.t. it) which is invariant under conjugation with unitaries and normalised so that its value on the identity operator is 1 .

We will see that, if $E$ lies in a gap then $\operatorname{IDS}(E)$ is a numerical topological invariant of the operator, which takes values in a countable sub-group of $\mathbb{R}$

\footnotetext{
${ }^{3}$ at low enough temperature
} 
which depends only on the underlying tiling. The topological invariance manifests itself in the property that the number is robust against perturbations which do not close the gap.

\subsection{Algebras for tilings}

We construct $C^{*}$-algebras for tilings for two reasons. First, by assigning in a coherent way a $C^{*}$-algebra to a tiling we can study the tiling using the tools of $C^{*}$-theory and non-commutative topology. Second, the algebra is a natural host for pattern equivariant operators for the tiling and so in particular contains operators describing the physics of the material modeled by the tiling.

The abstract definition of a $C^{*}$-algebra is as a complex $*$-Banach algebra whose norm satisfies the $C^{*}$-condition, $\left\|a^{*} a\right\|=\|a\|^{2}$. One of the fundamental theorems in $C^{*}$-theory says that any $C^{*}$-algebra can be faithfully represented on a Hilbert space. While this allows to view $C^{*}$-algebras more concretely as the norm closed sub-algebras of the bounded operators on a Hilbert space, a point of view which is close to physics, the abstract definition has its advantages when it comes to the study of its topological invariants.

\subsubsection{The pattern equivariant approach}

We describe the approach to tiling algebras via pattern equivariant integral kernels. When restricted to tilings of finite local complexity this is essentially the same than $[25,29]$.

We fix a tiling $\mathcal{T}$ which we view as the closed subset of $\mathbb{R}^{d}$ given by the boundary points of its tiles. We construct a $C^{*}$-algebra for $\mathcal{T}$ using double $R$-patch classes.

Recall that $\mathcal{L}^{R}(\mathcal{T})$ is the closure in the Hausdorff metric (between closed subsets of the $R$-ball at 0 ) of the set of all $R$-patches of $\mathcal{T}$ which are centered at 0 . Deemphazising the role of the origin we may also describe $\mathcal{L}^{R}(\mathcal{T})$ as the closure of $R$-patch classes of $\mathcal{T}$ in the Hausdorff metric, defining the Hausdorff distance between two equivalence classes as the infimum of the distance taken over all possible representatives. An element of the inverse limit $\Omega(\mathcal{T})=\lim _{+\infty \leftarrow R} \mathcal{L}^{R}(\mathcal{T})$ can then also be described as $[T ; x]$, the translational equivalence class of a tiling $T$ together with a point $x$ in the tiling. It corresponds to the tiling $T-x$, that is the representative of $T$ which has $x$ on the origin of $\mathbb{R}^{d}$.

Recall furthermore the definition of a double $R$-class of $\mathcal{T}$ as the equivalence class of a double $R$-patch $B_{R}[\mathcal{T} ; x, y]=B_{R}[\mathcal{T} ; x] \times B_{R}[\mathcal{T} ; y]$ under the diagonal action by translation. We denote the equivalence class by 
$\left[B_{R}[\mathcal{T} ; x, y]\right]$ and define, for $M \geq 0$

$$
\mathcal{L}^{R, M}(\mathcal{T})={\overline{\left\{\left[B_{R}[\mathcal{T} ; x, y]\right]:|y-x| \leq M\right\}}}^{d_{H}}
$$

the closure of the set of double $R$-classes of range bounded by $M$. Then $\mathcal{L}^{R}(\mathcal{T})=\mathcal{L}^{R, 0}(\mathcal{T})$, as we may identify $\left[B_{R}[\mathcal{T} ; x, x]\right]$ with $B_{R}[\mathcal{T}-x]$, the $R$-patch of $\mathcal{T}$ at $x$ shifted to 0

If $R>R^{\prime}$ we have an obvious surjection

$$
\mathcal{L}^{R, M}(\mathcal{T}) \rightarrow \mathcal{L}^{R^{\prime}, M}(\mathcal{T})
$$

given by $\left[B_{R}[\mathcal{T} ; x, y]\right] \mapsto\left[B_{R^{\prime}}[\mathcal{T} ; x, y]\right]$ and if $M<M^{\prime}$ an obvious inclusion

$$
\mathcal{L}^{R, M}(\mathcal{T}) \hookrightarrow \mathcal{L}^{R, M^{\prime}}(\mathcal{T})
$$

Consider the continuous function $v: \mathcal{L}^{R, M}(\mathcal{T}) \rightarrow B_{M}(0)$

$$
v\left(\left[B_{R}[T ; x, y]\right]\right)=x-y
$$

and define $C_{0}\left(\mathcal{L}^{R, M}(\mathcal{T})\right)$ to be the space of continuous functions (sliding block codes) $b: \mathcal{L}^{R, M}(\mathcal{T}) \rightarrow \mathbb{C}$ which vanish on $v^{-1}\left(\partial B_{M}(0)\right)$. Then the surjection (1.4) and the inclusion (1.5) induce inclusions

$$
C_{0}\left(\mathcal{L}^{R^{\prime}, M}(\mathcal{T})\right) \hookrightarrow C_{0}\left(\mathcal{L}^{R, M^{\prime}}(\mathcal{T})\right)
$$

where $R^{\prime}<R$ and $M<M^{\prime}$, which are algebra homomorphisms w.r.t. pointwise multiplication. We define the space of strongly pattern equivariant (for $\mathcal{T}$ ) elements as the algebraic direct limit

$$
A^{(s)}(\mathcal{T})=\lim _{R, M \rightarrow \infty} C_{0}\left(\mathcal{L}^{R, M}(\mathcal{T})\right)
$$

When equipped with pointwise multiplication and complex conjugation, $A^{(s)}(\mathcal{T})$ is a non-unital commutative $*$-algebra. But on $A^{(s)}(\mathcal{T})$ we have a second $*$-algebra structure, namely the non-commutative convolution product $C_{0}\left(\mathcal{L}^{R, M}(\mathcal{T})\right) \times C_{0}\left(\mathcal{L}^{R^{\prime}, M^{\prime}}(\mathcal{T})\right) \rightarrow C_{0}\left(\mathcal{L}^{R^{\prime \prime}, M^{\prime \prime}}(\mathcal{T}), R^{\prime \prime}=\max \left(R, R^{\prime}\right)\right.$, $M^{\prime \prime}=M+M^{\prime}$, defined by

$$
\begin{aligned}
b \star b^{\prime}\left(\left[B_{R^{\prime \prime}}[T ; x, y]\right]\right) & =\int_{B_{M}(x) \cap B_{M^{\prime}}(y)} b\left(\left[B_{R}[T ; x, z]\right]\right) b^{\prime}\left(\left[B_{R^{\prime}}[T ; z, y]\right]\right) d z \\
b^{*}\left(\left[B_{R}[T ; x, y]\right]\right) & =\overline{b\left(\left[B_{R}[T ; y, x]\right]\right)}
\end{aligned}
$$

Every element of $\Omega(\mathcal{T})$ defines a representation $\pi_{T}$ of $A^{(s)}(\mathcal{T})$ on $L^{2}\left(\mathbb{R}^{d}\right)$. Given $b \in C_{0}\left(\mathcal{L}^{R, M}(\mathcal{T})\right)$,

$$
\pi_{T}(b) \psi(x)=\int_{B_{M}(x)} b\left(\left[B_{R}[T ; x, z]\right]\right) \psi(z) d z .
$$


This is compatible with the inclusions and one easily sees that $\pi_{\mathcal{T}}$ is faithful, as the translates of $\mathcal{T}$ in $\Omega(\mathcal{T})$ form a dense set. As a result $\left\|\pi_{T}(b)\right\| \leq\left\|\pi_{\mathcal{T}}(b)\right\|$ for all $T \in \Omega(\mathcal{T})$. The continuous tiling algebra $A(\mathcal{T})$ is the completion of $\left(A^{(s)}(\mathcal{T}), \star\right)$ in the norm $\|b\|:=\left\|\pi_{\mathcal{T}}(b)\right\|$.

We may interprete the above in the following way: Any element $b \in$ $A^{(s)}(\mathcal{T})$ lies in some $C_{0}\left(\mathcal{L}^{R, M}(\mathcal{T})\right)$ and thus defines a strongly pattern equivariant function $f_{b}: \mathbb{R}^{d} \times \mathbb{R}^{d} \rightarrow \mathbb{C}$ by

$$
f_{b}(x, y)=b\left(\left[B_{R}[\mathcal{T} ; x, y]\right]\right)
$$

which we call its integral kernel. $A^{(s)}(\mathcal{T})$ can thus be seen as the algebra of strongly pattern equivariant integral kernels over $\mathbb{R}^{d}$ with their usual product and $*$-structure

$$
\begin{aligned}
f \star g(x, y) & =\int_{\mathbb{R}^{d}} f(x, z) g(z, y) d z \\
\left.f^{*}(x, y]\right) & =\overline{f(y, x)}
\end{aligned}
$$

and the representation $\pi_{\mathcal{T}}$ corresponds to the usual representation of integral kernels

$$
\pi_{\mathcal{T}}(b) \psi(x)=\int_{\mathbb{R}^{d}} f_{b}(x, z) \psi(z) d z
$$

Let $S$ be a Delone set which is locally derivable from $\mathcal{T}$. The discrete tiling algebra associated to $S$ is obtained by restricting the above construction to double $R$-patches $B_{R}[\mathcal{T} ; x, y]$ with $x, y \in S$. We denote $\mathfrak{L}_{S}^{R, M}(\mathcal{T}) \subset \mathcal{L}^{R, M}(\mathcal{T})$ the closure of the double $R$-patch classes with $x, y \in S$ and $\|y-x\| \leq M$. The discrete tiling algebra $\mathfrak{A}_{S}(\mathcal{T})$ is the completion of the algebra $\left(\mathfrak{A}_{S}^{(s)}(\mathcal{T}), \star\right)$ where

$$
\mathfrak{A}_{S}^{(s)}(\mathcal{T})=\lim _{R, M \rightarrow \infty} C_{0}\left(\mathfrak{L}_{S}^{R, M}(\mathcal{T})\right)
$$

is the algebraic limit and

$$
b \star b^{\prime}\left(\left[B_{R^{\prime \prime}}[T ; x, y]\right]\right)=\sum_{z \in S} b\left(\left[B_{R}[T ; x, z]\right]\right) b^{\prime}\left(\left[B_{R^{\prime}}[T ; z, y]\right]\right)
$$

and the $*$-structure is $b^{*}\left(\left[B_{R}[T ; x, y]\right]\right)=\overline{b\left(\left[B_{R}[T ; y, x]\right]\right)}$. Similar to the continuous case, every element of $\Xi(\mathcal{T})$ defines a representation $\pi_{T}^{S}$ of $A^{(s)}(\mathcal{T})$ on $L^{2}(S(T))$. Given $b \in C_{0}\left(\mathcal{L}^{R, M}(\mathcal{T})\right)$,

$$
\pi_{T}^{S}(b) \psi(x)=\sum_{z \in S(T) \cap B_{M}(0)} b\left(\left[B_{R}[T ; x, z]\right]\right) \psi(z)
$$

and the completion is taken in the norm $\|b\|=\left\|\pi_{\mathcal{T}}^{S}(b)\right\|$. The representation $\pi_{\mathcal{T}}^{S}$ on $\ell^{2}(S)$ can again be written using integral kernels, 


$$
\pi_{\mathcal{T}}^{S}(b) \psi(x)=\sum_{z \in S} f_{b}(x, z) \psi(z) .
$$

This corresponds exactly to (1.2) and indeed, tight binding operators with local coefficients are represented by elements of the discrete tiling algebra.

One advantage of discrete tiling algebras is that they are unital. Indeed, since $S$ is uniformly discrete, $C_{0}\left(\mathfrak{L}_{S}^{R, M}(\mathcal{T})\right)=C\left(\mathfrak{L}_{S}^{R, 0}(\mathcal{T})\right)$ for some small enough $M>0$. Therefore $\mathfrak{A}_{S}^{(s)}(\mathcal{T})$ contains $C\left(\mathfrak{L}_{S}^{R, 0}(\mathcal{T})\right)$ and thus a unit.

\subsubsection{The groupoid approach}

While the definition of the tiling algebras by means of pattern equivariant functions is very intuitive, as it mimicks local operators, a more abstract definition is needed for $K$-theory calculations. In fact, the algebras can be understood as groupoid $C^{*}$-algebras and in the case that the groupoid is a transformation groupoid, they become crossed product algebras for which tools in $K$-theory have been developped.

The Gelfand spectrum of the commutative algebra $A^{(s)}(\mathcal{T})$ with pointwise multiplication is given by

$$
\mathcal{G}(\mathcal{T})=\bigcup_{M \geq 0} \lim _{\infty \leftarrow R} \mathcal{L}^{R, M}(\mathcal{T})
$$

The elements of $\mathcal{G}$ are of the form $[T ; x, y]$ where $[T, x] \in \Omega(\mathcal{T}) . \mathcal{G}$ is a the continuous tiling groupoid, it carries the groupoid product

$$
[T ; x, y]\left[T^{\prime} ; x^{\prime}, y^{\prime}\right]=\left[T, x, y^{\prime}\right] \quad \text { provided } T^{\prime}=T \text { and } x^{\prime}=y \text {. }
$$

$C_{c}(\mathcal{G})$ with the standard convolution product for groupoid algebras is nothing else then $\left(A^{(s)}(\mathcal{T}), \star\right) . A(\mathcal{T})$ is therefore the groupoid $C^{*}$-algebra of $\mathcal{G}(\mathcal{T})$.

Under the homeomorphism $\mathcal{G}(\mathcal{T}) \ni[T ; x, y] \mapsto([T ; x], x-y) \in \Omega(\mathcal{T}) \times \mathbb{R}^{d}$ the product becomes

$$
\left.([T ; x], v)\left(\left[T^{\prime}, x^{\prime}\right], v^{\prime}\right]\right)=\left([T ; x], v+v^{\prime}\right) \quad \text { provided }\left[T^{\prime} ; x^{\prime}\right]=[T, x-v]
$$

The product encodes thus the action $\alpha_{v}([T ; x]):=[T ; x-v]$ of $\mathbb{R}^{d}$ on $\Omega(\mathcal{T})$. It follows that the continuous tiling algebra is the crossed product $C^{*}$-algebra

$$
A(\mathcal{T}) \cong C(\Omega(\mathcal{T})) \rtimes_{\alpha} \mathbb{R}^{d}
$$

associated to the continuous dynamical system $\left(\Omega(\mathcal{T}), \mathbb{R}^{d}, \alpha\right)$ of the tiling.

Also $\mathfrak{A}_{S}(\mathcal{T})$ can be interpreted as a groupoid $C^{*}$-algebra, namely of the discrete tiling groupoid associated to $S$, 


$$
\mathfrak{G}_{S}(\mathcal{T})=\bigcup_{M \geq 0} \lim _{\infty \leftarrow R} \mathfrak{L}_{S}^{R, M}(\mathcal{T})
$$

whose unit space is the discrete hull

$$
\Xi_{S}=\lim _{\infty \leftarrow R} \mathfrak{L}_{S}^{R, 0}(\mathcal{T})
$$

Indeed, the elements of $\mathfrak{G}_{S}$ are given by triples $[T ; x, y]$ with $x, y \in S(T)$ and $T \in \Omega(\mathcal{T})$ and the product is the same as for $\mathcal{G}$ but $x, y$ are restricted to $S(T)$.

\subsubsection{Crossed products with $\mathbb{Z}^{d}$}

The case which we will describe in more detail is the one in which $\mathfrak{G}_{S}(\mathcal{T})$ is actually a transformation groupoid so that $\mathfrak{A}_{S}(\mathcal{T})$ is a crossed product by $\mathbb{Z}^{d}$. As some details about crossed products with $\mathbb{Z}^{d}$ are needed further down we recall them here, see also [15]. For that we start more generally with an action $\alpha$ of $\mathbb{Z}^{d}$ on a (unital) $C^{*}$-algebra $B$. Let $B_{\alpha} \mathbb{Z}^{d}$ be the linear space of Laurent polynomials $\sum_{n \in \mathbb{Z}^{d}} b_{n} u^{n}$ (only finitely many $b_{n}$ are non-zero) with coefficients $b_{n} \in B$. Here $u_{1}, \cdots, u_{d}$ are $d$ variables and $u^{n}=u_{1}^{n_{1}} \cdots u_{d}^{n_{d}}$. Define the associative product and $*$-structure

$$
b_{n} u^{n} b_{m}^{\prime} u^{m}=b_{n} \alpha_{n}\left(b_{m}^{\prime}\right) u^{n+m}, \quad\left(b u^{n}\right)^{*}=\alpha_{-n}\left(b^{*}\right) u^{-n} .
$$

In particular, the $u_{1}, \cdots, u_{d}$ commute and satisfy the relations of unitaries, $u_{i} u_{i}^{*}=u_{i}^{*} u_{i}=1$.

Any representation $\rho$ of $B$ on some Hilbert space $\mathcal{H}$ gives rise to a representation $\pi_{\rho}$ of $B_{\alpha} \mathbb{Z}^{d}$ on $\ell^{2}\left(\mathbb{Z}^{d}, \mathcal{H}\right)$ :

$$
\pi_{\rho}(b) \psi(n)=\rho\left(\alpha_{-n}(b)\right) \psi(n), \quad \pi_{\rho}\left(u^{m}\right) \psi(n)=\psi(n-m)
$$

and if $\rho$ is faithful then the crossed product $C^{*}$-algebra $B \rtimes_{\alpha} \mathbb{Z}^{d}$ is the completion of the above algebra $B_{\alpha} \mathbb{Z}^{d}$ w.r.t. the norm $\|\cdot\|:=\left\|\pi_{\rho}(\cdot)\right\| \cdot \pi_{\rho}$ is called the representation induced by $\rho$.

We now specify to the case of discrete tiling algebras which may be written as crossed products with $\mathbb{Z}^{d}$. This might not always be the case and so we need to make some assumptions. We say that $\mathcal{T}$ is a decoration of $\mathbb{Z}^{d}$ if there exists an oriented graph $\Gamma$, such that

1. There is an isomorphism (of oriented graphs) $\nu$ between $\Gamma$ and the Caley graph of $\mathbb{Z}^{d}$,

2. the set of vertices $S$ of $\Gamma$ is a Delone subset of $\mathbb{R}^{d}$ which is locally derivable from $\mathcal{T}$, 
3. for all edges $(x, y)$ of $\Gamma, \nu(x, y)$ is locally derivable from $\mathcal{T}$,

4. the combinatorial distance between $x, y \in S$ (the minimal number of edges needed to link $x$ with $y$ ) is Lipschitz equivalent to their euclidean distance in $\mathbb{R}^{d}$.

Before we exploit these conditions we remark that they naturally occur in many situations. They are clearly satisfied for Wang tilings where we can take $S$ to be the vertices and the edges of $\Gamma$ to be the edges of the cubes. The orientation can be coherently fixed by a global choice of orientation on $\mathbb{R}^{d}$. More generally, an tiling from which we can locally derive a lattice $S$ allows the construction of such a graph $\Gamma$ in a similar way. Recall that after deformation this can always be achieved for FLC tilings. But $S$ does not have to be a lattice, for instance, for one dimensional tilings (with a lower and an upper bound on thel tile size) we may use the tiles to define the edges of $\Gamma$, oriented according to a choice of orientation for $\mathbb{R}$.

Let $\nu$ be an isomorphism between $\Gamma$ and the Caley graph of $\mathbb{Z}^{d}$. Then we can define a map

$$
\tilde{v}: \mathfrak{G}_{S}(\mathcal{T}) \rightarrow \mathbb{Z}^{d}
$$

as follows. Given $(x, y) \in S \times S$ there is a path in $\Gamma$ from $y$ to $x$. We map this path with $\nu$ to a path in the Caley graph of $\mathbb{Z}^{d}$. As such the path corresponds to an element of $\mathbb{Z}^{d}$ which we denote $\nu(x, y)$. While the path need not be unique, the group element is. We now define

$$
\tilde{v}([\mathcal{T} ; x, y])=\nu(x, y) .
$$

By the above conditions 2. and 3., for any edge $(x, y)$ of $\Gamma$ there exists $R>0$ such that $\tilde{v}[\mathcal{T} ; x, y])$ can be derived from the double $R$-patch class $\left[B_{R}[\mathcal{T} ; x, y]\right]$. The $R$ depends on $(x, y)$ but since, by 4 ., the combinatorial distance between $x$ and $y$ is bounded by a constant times their euclidean distance, we can, given $M>0$, choose the $R$ uniformly for all $(x, y) \in S \times S$ with $\|y-x\| \leq M$. This shows that $\tilde{v}$ is extends by continuity to $\mathfrak{G}_{S}(\mathcal{T})$. From $\nu(x, y)=\nu(x, z)+\nu(z, y)$ for all $z \in S$ we derive that $\tilde{v}$ is a groupoid homomorphism. Clearly, when restricted to the orbit of $[\mathcal{T} ; x, x]$ in $\mathfrak{G}_{S}(\mathcal{T})$ (which is a group), then $\tilde{v}$ is a group isomorphism. Since the orbit of $\mathcal{T}$ is dense by construction, the restriction of $\tilde{v}$ to any orbit of $\mathfrak{G}_{S}(\mathcal{T})$ is an isomorphism. We therefore can define an action $\alpha$ on $\Xi_{S}$ by $\mathbb{Z}^{d}$,

$$
\alpha_{n}([T ; x])=[T, y]
$$

where $y$ is the unique element of $S$ such that $\tilde{v}([T ; x, y])=n$. Since $\tilde{v}$ is strongly pattern equivariant this action is continuous and the map

$$
\mathfrak{G}_{s} \ni[T ; x, y] \mapsto([T ; x], \tilde{v}([T ; x, y])) \in \Xi_{S} \rtimes_{\alpha} \mathbb{Z}^{d}
$$

a continuous groupoid homorphism. Moreover, the map is bijective. Condition 4 now garantees that its inverse is continuous. Indeed, it implies that 
$\tilde{v}^{-1}\left(e_{i}\right) \in \mathfrak{L}_{S}^{R, M}$ for some finite $R$ and $M$, where $e_{i}$ is a generator of $\mathbb{Z}^{d}$. Since $\mathfrak{L}_{S}^{R, M}$ is compact also $\tilde{v}^{-1}\left(e_{i}\right)$ is compact, which shows that the restriction of $\tilde{v}$ to $\tilde{v}^{-1}\left(e_{i}\right)$ is a homeomorphism onto $\Xi_{S} \times\left\{e_{i}\right\}$. A similar argument works for any $n \in \mathbb{Z}^{d}$.

It now follows from the general theory [37] that the discrete tiling algebra $\mathfrak{A}_{S}(\mathcal{T})$ is isomorphic to the crossed product $C\left(\Xi_{S}\right) \rtimes_{\alpha} \mathbb{Z}^{d}$ of $C\left(\Xi_{S}\right)$ with $\mathbb{Z}^{d}$. We find it instructive to explain this directly: We have seen that $\mathfrak{A}_{S}^{(s)}(\mathcal{T})$ contains $C\left(\mathfrak{L}_{S}^{R, 0}(\mathcal{T})\right)$. It furthermore contains the indicator functions on compact open subsets of $\mathfrak{L}_{S}^{R, 0}(\mathcal{T})$. Above we saw that $\tilde{v}^{-1}(n)$ is compact. It is also open and thus its indicator function $\chi_{n}$ an element of $\mathfrak{A}_{S}(\mathcal{T})$. Let $b \in C\left(\mathfrak{L}_{S}^{R, 0}(\mathcal{T})\right.$. Then

$\chi_{n} \star b([T ; x, y])=\chi_{n}([T ; x, y]) b([T ; y, y]), \quad b \star \chi_{n}([T ; x, y])=b([T ; x, x]) \chi_{n}([T ; x, y])$.

Now $\chi_{n}([T ; x, y])=1$ is equivalent to $\alpha_{n}(b)([T ; x, x])=b([T ; y, y])$ and hence we have $\chi_{n} \star b=\alpha_{n}(b) \star \chi_{n}$. Therefore, if $u^{n}=\chi_{n}$, the relations (1.7) are satisfied. This shows that $\mathfrak{A}_{S}^{(s)}(\mathcal{T})$ contains $B_{\alpha} \mathbb{Z}^{d}$ with $B=\lim _{R \rightarrow \infty} C\left(\mathfrak{L}_{S}^{R, 0}(\mathcal{T})\right)$. Furthermore, any $b \in C_{0}\left(\mathfrak{L}_{S}^{R, M}(\mathcal{T})\right.$ can be written as a finite linear combination of elements from $C\left(\mathfrak{L}_{S}^{R, 0}(\mathcal{T})\right.$ and $\chi_{n}$. Hence $B_{\alpha} \mathbb{Z}^{d}$ is dense in $\mathfrak{A}_{S}(\mathcal{T})$. Since $C\left(\Xi_{S}\right)$ is the closure of $B$ we have $\mathfrak{A}_{S}(\mathcal{T}) \cong C\left(\Xi_{S}\right) \rtimes_{\alpha} \mathbb{Z}^{d}$.

Given $[T ; x] \in \Xi_{S}$ the representation $\pi_{T}^{S}(1.6)$ is unitarily equivalent to a representation on $\ell^{2}\left(\mathbb{Z}^{d}\right)$ in which $\chi_{n}$ acts as the translation operator $T^{n}$. Indeed, the unitary intertwining the representation corresponds to the bijection $\nu(x, \cdot): S(T-x) \rightarrow \mathbb{Z}^{d}$. Furthermore, the representation on $\ell^{2}\left(\mathbb{Z}^{d}\right)$ obtained in this way is the $d$-fold iterated induced representation of the representation $\rho_{T}: C\left(\Xi_{S}\right) \rightarrow \mathbb{C}$ given by $\rho_{T}(f) \psi(x)=f([T ; x]) \psi(x)$.

For Wang tilings, the symbolic hull $\Xi$ plays the role of $\Xi_{S}$.

\subsubsection{Extensions and exact sequences}

One of the basic building blocks of algebraic topology are extentions. An extension of a $C^{*}$-algebra $A$ is a $C^{*}$-algebra $E$ together with a sujective morphism $q: E \rightarrow A$. Let $J=\operatorname{ker} q . J$ is a closed two-sided ideal of $E$. Denoting by $i: J \rightarrow E$ the inclusion map we have a short exact sequence (SES)

$$
0 \rightarrow J \stackrel{i}{\rightarrow} E \stackrel{q}{\rightarrow} A \rightarrow 0
$$

that is, a chain of algebras with morphisms $i, q$ such that $i$ is injective, $\operatorname{im} i=\operatorname{ker} q$ and $q$ is surjective.

Of particular importance is the Toeplitz extension $\mathcal{T}(B, \beta)$ of the crossed product $A=B \rtimes_{\beta} \mathbb{Z}$. Its construction is close to that of $B \rtimes_{\beta} \mathbb{Z}$, but instead of the algebra $B_{\beta} \mathbb{Z}$ generated by the elements of $B$ and a unitary $u$ subject 
to the relation $u b=\beta(b) u$ we now take the algebra generated by the elements of $B$ and a (proper) isometry $\hat{u}$ and its $*$-adjoint $\hat{u}^{*}$, which are subject to the relations

$$
\hat{u} b=\beta(b) \hat{u}, \quad b \hat{e}=\hat{e} b
$$

where $\hat{e}=1-\hat{u} \hat{u}^{*}$, and $b \in B$. Saying that $\hat{u}$ is an isometry means that $\hat{u}^{*} \hat{u}=1$ and implies that $\hat{e}$ is a projection, properness requires $\hat{e}$ to be neither 0 nor 1 . We denote this algebra by $B_{\beta} \mathbb{N}$. Elements of $B_{\beta} \mathbb{N}$ are thus finite sums of finite products of elements from $B, \hat{u}$ and $\hat{u}^{*}$. The above relation imply that also $\hat{u}^{*} b=\beta^{-1}(b) \hat{u}^{*}$.

We have a sujective $*$-algebra morphism $q: B_{\beta} \mathbb{N} \rightarrow B_{\beta} \mathbb{Z}$ which is given by

$$
q(b)=b, \quad q(\hat{u})=u
$$

and hence satisfies $q(\hat{e})=0$. The kernel of $q$ is the subalgebra generated by $\hat{e}$. Identifying $\hat{e}$ with the half-infinite Jacobi matrix which has as only non-zero entry a 1 at the upper left corner, and $\hat{u}$ with the Jacobi matrix which is 1 on the lower diagonal, we see that $\operatorname{ker} q$ can be identified with the algebra of half-infinite matrices with entries from $B$ of which only finitely many are non-zero. This is the tensor product $B \otimes F$ where $F$ is the algebra of halfinfinite complex matrices with only finitely many are non-zero entries. We thus have a SES

$$
0 \rightarrow B \otimes F \rightarrow B_{\beta} \mathbb{N} \stackrel{q}{\rightarrow} B_{\beta} \mathbb{Z} \rightarrow 0
$$

The Toeplitz extension is the universal $C^{*}$-completion of $B_{\beta} \mathbb{N}$ and gives rise to the SES of $C^{*}$-algebras

$$
0 \rightarrow B \otimes \mathcal{K} \rightarrow \mathcal{T}(B, \beta) \stackrel{q}{\rightarrow} B \rtimes_{\beta} \mathbb{Z} \rightarrow 0
$$

Here $\mathcal{K}$ is the algebra of compact operators. Recall that a representation $\rho$ of $B$ on $\mathcal{H}$ induces a representation $\pi_{\rho}$ of $B \rtimes_{\beta} \mathbb{Z}$ on $\ell^{2}(\mathbb{Z}, \mathcal{H})$. It also induces a representation $\hat{\pi}_{\rho}$ of $\mathcal{T}(B, \beta)$ on $\ell^{2}(\mathbb{N}, \mathcal{H})$, namely

$$
\pi_{\rho}(b) \psi(n)=\rho\left(\alpha_{-n}(b)\right) \psi(n), \quad \pi_{\rho}(\hat{u}) \psi(n)=\psi(n-1) \text { with } \psi(-1)=0 .
$$

\section{$1.4 K$-theoretic invariants for tilings}

Having assigned to a tiling various $C^{*}$-algebras, their $K$-groups yield invariants for the tiling. These invariants can serve to distinguish tilings, or even classify them under weaker notions of equivalence. But they also aquire physical significance, as the algebra is physically motivated.

In turns out that the discrete tiling algebras constructed above for a given tiling and a Delone set $S$ which is locally derivable from the tiling are all strongly Morita equivalent to the continuous tiling algebra. Since $K$-groups do not distinguish strongly Morita equivalent algebras, the different algebras 
lead to the same $K$-groups. Having said that, $K$-groups can come with extra structure. The $K_{0}$-group can be ordered, for instance, and scaled (this is then called a dimension group) [39]. Whereas the order structure is again the same for strongly Morita equivalent algebras, the scale is not, and different choices for $S$ lead to different scales.

\subsubsection{Short definition of $K$-theory}

We provide a definition of the $K$-groups which is motivated by physics. The elements of the groups are essentially homotopy classes of Hamiltonians which have a gap in their spectrum. This approach is based on van Daele's formulation of $K$-theory [12] and has been adapted to topological phases in [32], though we consider only the complex case here. For the more traditional approach we refer the reader to $[39,9]$.

If the local arrangement of atoms in a solid are described by a tiling (in the sense of being mutually locally derivable with the tiling) then it is natural to consider the tiling algebra as the $C^{*}$-algebra of observables. But what follows can in principle be carried out with any $C^{*}$-algebra $A$ which is motivated by the physical system one wants to consider.

The energy observable corresponds to a self-adjoint element $H$ of $A$ (the Hamiltonian, or a bounded function of the Hamiltonian), represented in the relevant physical representation of $A$. A gap in the spectrum of $H$ indicates that the system is an insulator, provided the Fermi energy lies in that gap. The topological phase of the insulator is, by definition, the homotopy class of $H$ under continuous deformation which preserves self-adjointness and the presence of a gap at the Fermi energy. Assuming that $A$ is unital we may shift the gap and the Fermi energy to 0 and thus say that the topological phase of $H$ is its connected component in the set of self-adjoint invertible elements $G L\left(A^{\text {s.a. }}\right)$ of $A$. Following a standard procedure one can construct an abelian group $K_{0}(A)$ whose elements are generated by the homotopy classes of $G L\left(A^{\text {s.a. }}\right) . K_{1}(A)$ is obtained by a similar construction, but for the set of connected components of $G L(A)$, that is, without the self-adjointness constreint. In physics, the role of an invertible element $Q \in A$ is as the chiral half of an insulator with chiral symmetry; the Hamiltonian of such an insulator has the form $H=\left(\begin{array}{cc}0 & Q^{*} \\ Q & 0\end{array}\right)$.

To construct a group out of the homotopy classes of $G L(A)$ we consider homotopy classes in all $G L\left(M_{n}(A)\right), n \geq 1$, the invertible elements in the matrix algebras over $A$. To compare matrices of different sizes we use the inclusion $G L\left(M_{n}(A)\right) \ni x \mapsto x \oplus 1 \in G L\left(M_{n+1}(A)\right)$ which induces an inclusion of homotopy classes. The direct limit of the quotient spaces by homotopy $\lim _{n} G L\left(M_{n}(A)\right) / \sim_{h}$ admits an addition $[x]+[y]=[x \oplus y]([x]$ denotes the homotopy class of $x$ ). It turns out that $x \oplus y$ is homotopic to $x \oplus y$ in 
$G L\left(M_{2}(A)\right)$, and also to $x y \oplus 1$. The addition law defined above is therefore the abelianization of the product in $A$ and yields an abelian group structure with neutral element $[1]$ and inverse $-[x]=\left[x^{-1}\right] . K_{1}(A)$ is this abelian group.

The construction of $K_{0}(A)$ follows similar lines, except one requires selfadjointness, and therefore considers the direct $\operatorname{limit}_{n} \lim _{n} G L\left(M_{n}(A)^{s . a .}\right) / \sim_{h}$. Addition is still defined by $[x]+[y]=[x \oplus y]$, but since a product of two selfadjoint elements need not to be self-adjoint, it is not the abelianization of the product in $A$ and $V(A):=\lim _{n} G L\left(M_{n}(A)^{s . a .}\right) / \sim_{h}$ does not contain inverse elements. Instead $V(A)$ is an abelian monoid with neutral element [1]. $K_{0}(A)$ is the associated Grothendieck group $V(A) \times V(A) / \sim$. Here $\left(\left[x_{1}\right],\left[y_{1}\right]\right) \sim$ $\left(\left[x_{2}\right],\left[y_{2}\right]\right)$ if there exist $[z] \in V(A)$ such that $\left[x_{1}\right]+\left[y_{2}\right]+[z]=\left[x_{2}\right]+\left[y_{1}\right]+[z]$ and addition is componentwise, $\left(\left[x_{1}\right],\left[y_{1}\right]\right)+\left(\left[x_{2}\right],\left[y_{2}\right]\right)=\left(\left[x_{1}\right]+\left[x_{2}\right],\left[y_{1}\right] \oplus\right.$ $\left.\left[y_{2}\right]\right)$.

The more conventional picture of $K_{0}(A)$ uses homotopy classes of projections. Any $H \in G L\left(A^{s . a .}\right)$ is homotopic to a self-adjoint unitary, that is, an element of the form $1-2 p$ where $p$ is a projection. This projection is the spectral projection onto the negative spectral part of $H$. A homotopy class of invertible self-adjoint elements corresponds therefore to a homotopy class of projections.

Similarily, any invertible element is homotopic to a unitary. This yields the conventional picture of $K_{1}(A)$ as homotopy classes of unitaries.

\subsubsection{Basic properties of $K$-theory}

We list the basic properties of $K_{0}$ and $K_{1}$.

1. Functoriality. Whenever we have an algebra homomorphism $\varphi: A \rightarrow$ $B$ between two unital $C^{*}$-algebras and $\varphi$ preserves the unit, then we obtain a group homomorphism $\varphi_{*}: K_{i}(A) \rightarrow K_{i}(B)$ simply by setting $\varphi_{*}([x])=[\varphi(x)]$ where $\varphi$ is extended to $M_{n}(A)$ entrywise. Furthermore $(\psi \circ \varphi)_{*}=\psi_{*} \circ \varphi_{*}$.

2. $K_{i}$ preserves direct sums. $K_{i}(A \oplus B)=K_{i}(A) \oplus K_{i}(B)$.

3. Stability. The map $x \mapsto x \oplus 1$ induces an isomorphism $K_{i}\left(M_{n}(A)\right) \rightarrow$ $K_{i}\left(M_{n+1}(A)\right)$. In particular, $K_{i}(\mathcal{K} \otimes A) \cong K_{i}(A)$, where $\mathcal{K}$ are the compact operators, and (strongly) Morita equivalent algebras have the same $K$-theory.

4. Continuity. $K_{i}$ commutes with direct limits, $K_{i}\left(\lim _{n} A_{n}\right)=\lim _{n} K_{i}\left(A_{n}\right)$.

\subsubsection{Definition of $K_{i}(A)$ for non-unital algebras}

The definition we gave above for $K_{i}(A)$ needs invertibility, and hence a unit for $A$. If $A$ is not unital we have to add a unit to $A$. This is done in the following way. 
Let $A^{+}=A \times \mathbb{C}$ with product $(a, \lambda)(b, \mu)=(a b+\lambda b+\mu a, \lambda \mu)$ and $*-$ structure $(a, \lambda)^{*}=\left(a^{*}, \bar{\lambda}\right)$. This is a unital algebra with unit $(0,1)$. We have a surjective algebra homomorphism $\pi: A^{+} \rightarrow \mathbb{C}, \pi(a, \lambda)=\lambda$, whose kernel is $A$. Since $A^{+}$is unital we may define its $K_{i}$-groups in the above way $\left(A^{+}\right.$ is a $C^{*}$-algebra in a natural way). We thus get a group homomorphism $\pi_{*}$ : $K_{i}\left(A^{+}\right) \rightarrow K_{i}(\mathbb{C})$. By definition,

$$
K_{i}(A)=\operatorname{ker} \pi_{*}
$$

\subsubsection{More involved properties of $K$-theory}

The definition, in particular of $K_{0}(A)$ for non-unital $A$ is not very direct, but it is crucial to obtain the following important properties.

1. Bott periodicity. We have not given a definition of $K_{i}(A)$ for $i>1$, but the reasonable definition of $K_{i}(A)$ for $i$ even, or odd, turns out to be isomorphic to $K_{0}(A)$, or $K_{1}(A)$, respectively. This is called Bott periodicity.

2. Six term exact sequence (boundary maps). Given a short exact sequence of $C^{*}$-algebras

$$
0 \rightarrow J \stackrel{i}{\rightarrow} E \stackrel{q}{\rightarrow} A \rightarrow 0
$$

there are group homomorphisms (boundary maps) $\delta_{i}: K_{i}(A) \rightarrow K_{i-1}(J)$ making the following long sequence of $K$-groups exact

$$
K_{i}(J) \stackrel{i_{*}}{\rightarrow} K_{i}(E) \stackrel{q_{*}}{\rightarrow} K_{i}(A) \stackrel{\delta_{i}}{\rightarrow} K_{i-1}(J) \cdots
$$

Due to Bott periodicity, the above sequence is of period 6 . We will provide formulas for the boundary maps below which can be interpreted physically.

\subsubsection{Calculating $K$-groups for tiling algebras}

There are tools to calculate the $K$-groups for the continuous tiling algebra $C(\Omega) \rtimes \mathbb{R}^{d}$ and tools to calculate them in the case there is a transversal $\Xi_{S}$ such that the corresponding discrete tiling algebra is a crossed product $C\left(\Xi_{S}\right) \rtimes_{\alpha} \mathbb{Z}^{d}$. We saw that the latter is garanteed if the tiling has FLC. Apart from the scale, the $K$-groups of $C(\Omega) \rtimes \mathbb{R}^{d}$ and $C\left(\Xi_{S}\right) \rtimes_{\alpha} \mathbb{Z}^{d}$ coincide, as the algebras are strongly Morita equivalent. Both calculations need extra structure and boil down to express the $K$-groups as cohomology groups. 


\subsubsection{Via the Connes-Thom isomorphism and approximants}

One method of computation of their $K$-groups makes use of the approximants $\mathcal{L}^{R}(\mathcal{T})$ of the tiling discussed above. This method is feasible if the approximates are subject to a strong hierarchical order, as, for instance, substitutional tilings $[1,29,41]$.

The Connes-Thom isomorphism [11] is one of the fundamental results in $K$-theory which allows to relate the $K$-theory of a crossed product algebra with $\mathbb{R}, B \rtimes_{\beta} \mathbb{R}$, to the $K$-theory of $B$. Indeed, for any (continuous) action $\beta$ of $\mathbb{R}$ on a $C^{*}$-algebra $B$ one has

$$
K_{i}\left(B \rtimes_{\beta} \mathbb{R}\right) \cong K_{i-1}(B) .
$$

Starting with the continuous algebra $C(\Omega) \rtimes_{\alpha} \mathbb{R}^{d}$ we can apply iteratively the Connes-Thom isomorphism to get

$$
K_{i}\left(C(\Omega) \rtimes_{\alpha} \mathbb{R}^{d}\right) \cong K_{i-d}(C(\Omega)) .
$$

As $\Omega(\mathcal{T})=\lim \mathcal{L}^{R}(\mathcal{T})$ the algebra $C(\Omega)$ is the $C^{*}$-completion of $\lim C\left(\mathcal{L}^{R}(\mathcal{T})\right)$ so that, using the continuity of the $K$-functor we get

$$
K_{i}(C(\Omega))=\lim _{R \rightarrow+\infty} K_{i}\left(C\left(\mathcal{L}^{R}\right)\right)
$$

The $\mathcal{L}^{R}$ are flat branched manifolds. For low enough dimension $(d \leq 3)$ $K_{i}\left(C\left(\mathcal{L}^{R}\right)\right)$ is isomorphic to the singular cohomology of the space $\mathcal{L}^{R}$ which is computable. If $\mathcal{T}$ has a hierarchical structure relating $\mathcal{L}^{R}$ for different $R$ (or suitable modifications thereof) then the direct limit can be computed. For substitution tilings a computer code is available to perform these calculations $[18]$.

\subsubsection{Via iteration of the Pimsner-Voiculescu sequence and group cohomology}

A second approach to the calculation of $K$-groups of tilings which are decorations of $\mathbb{Z}^{d}$ is based on the iteration of the Pimsner-Voiculescu sequence [34]. It is useful if one has good control on the action $\alpha$ of $\mathbb{Z}^{d}$ on the discrete hull $\Xi[17,22,19,24]$.

Consider an isomorphism $\phi: M \rightarrow M$ of an abelian group. The quotient $C_{\phi} M:=\operatorname{coker}(\mathrm{id}-\phi)$ is called the group of coinvariants and the subgroup $I_{\phi} M:=\operatorname{ker}(\mathrm{id}-\phi)$ the group of invariants of $M$ w.r.t. to $\phi$. These are the cohomology groups of the group $\mathbb{Z}$ with coefficients in $M$, namely $H^{0}(\mathbb{Z}, M)=$ $I_{\phi} M$ and $H^{1}(\mathbb{Z}, M)=C_{\phi} M$. Any isomorphism $\beta$ on $B$ induces a isomorphism $\beta_{*}$ on $K_{i}(B)$. 
The Toeplitz extension (1.8) gives rise to a 6 -term exact sequence, the Pimsner-Voiculescu sequence, which can be split into two SES $(i=1,2)$

$$
0 \rightarrow C_{\beta_{*}} K_{i}(B) \stackrel{\iota_{*}}{\rightarrow} K_{i}\left(B \rtimes_{\beta} \mathbb{Z}\right) \stackrel{\delta_{i}}{\rightarrow} I_{\beta_{*}} K_{i-1}(B) \rightarrow 0 .
$$

where $\imath_{*}$ is induced by the inclusion $B \hookrightarrow B \rtimes_{\beta} \mathbb{Z}$ and $\delta_{i}$ is the boundary map from Section 1.4.1.3. By iteration we can express the $K$-group of $C(\Xi) \rtimes_{\alpha} \mathbb{Z}^{d}$ through similar SESs with coinvariants and invariants of $K_{i}(C(\Xi))$. If $d \leq 3$ then all these sequence split and we obtain $[22]$

$$
K_{i}\left(C(\Xi) \rtimes_{\alpha} \mathbb{Z}^{d}\right) \cong \bigoplus_{k} H^{i+2 k}\left(\mathbb{Z}^{d}, C(\Xi, \mathbb{Z})\right)
$$

The group cohomology is computable if we have a good understanding of the $\mathbb{Z}^{d}$-action $\alpha$ on $\Xi$. This is, for instance, the case for almost canonical cut \& project tilings, and also here computer codes are available to perform the calculations [18].

\subsubsection{Numerical invariants for pattern equivariant operators}

We have seen that a pattern equivariant operator is an element in the representation $\pi_{\mathcal{T}}$, or $\pi_{\mathcal{T}}^{S}$, of the continuous or discrete algebra of some tiling $\mathcal{T}$. We denote this algebra now simply by $A$.

If the operator is self-adjoint and invertible it comes from a self-adjoint invertible element $h \in A$ and so defines a class in $K_{0}(A)$. This class is the the equivalence class of $([h],[1]) \in V(A) \times V(A)$ under the relation $\sim$ defining the Grothendieck group which we discussed above, but we simply denote this class by $[h]$, as the further calculations depend essentially only on the homotopy class of $h$.

If the operator is only invertible thus coming from an invertible element $Q \in A$ then its homotopy class defines an element in $K_{1}(A)$.

The $K$-classes are the abstract topological invariants of the operators. They are invariants in the sense that they are stable under continuous deformation in $G L\left(A^{\text {s.a. }}\right)$ or $G L(A)$, respectively.

Numerical topological invariants for pattern equivariant operators can now be obtained from additive functionals on $K_{i}(A)$. Such functionals can be given by index pairings with $K$-homology classes of $A$ [21], or by Chern characters [11]. We will discuss here only the simplest cases, namely the functional on $K_{0}(A)$ defined by a trace, and the winding number on $K_{1}(A)$ defined by a trace and a derivation. These have physical interpretation in the examples we consider below. 


\subsubsection{Traces and gap labelling}

Gap-labelling means the assignment of labels to the gaps in the spectrum of a self-adjoint operator. These labels are supposed to be stable under continuous deformations of the operator which do not close the gap. In Bellissard's approach to the gap-labelling by means of the $K_{0}$-group of the $C^{*}$-algebra $A$ of observables $[6,7]$ the operator is an element of $A$ and continuity means continuity in the norm of that algebra.

A (positive) trace on $A$ is a linear functional tr $: A \rightarrow \mathbb{C}$ which satisfies $\operatorname{tr}\left(u a u^{*}\right)=\operatorname{tr}(a)$ for all $a \in A$ and unitary $u \in A^{+}$, and $\operatorname{tr}\left(a^{*} a\right) \geq 0$. It follows that $\operatorname{tr}(p)=\operatorname{tr}\left(p^{\prime}\right)$ for two homotopic projections. Let $P_{-}(h)$ be the spectral projection onto the negative energy part of $h$. Note that $P_{-}(h) \in A$ since $h$ has a spectral gap at energy 0 . Thus $h \mapsto \operatorname{tr}\left(P_{-}(h)\right)$ is homotopy invariant in $G L\left(A^{s . a .}\right)$ and

$$
\operatorname{tr}_{*}([h])=\operatorname{tr}\left(P_{-}(h)\right)
$$

extends to a functional on $K_{0}(A)$ with values in $\mathbb{R}$. Indeed, if $h \in G L\left(M_{n}(A)^{\text {s.a. }}\right)$ we extend tr to $M_{n}(A)$ using the matrix trace.

We can therefore formulate the gap labelling in the following way: If $h$ is a self-adjoint element of $A$ and $\Delta$ a gap in the spectrum of $h$ then, for any $E \in \Delta$ the shifted element $h-E$ belongs to $G L\left(A^{s . a .}\right)$ and so defines an element of $K_{0}(A)$. This class $[h-E]$ is the abstract gap-label of the gap $\Delta$ of $h$.

If we have a trace $\operatorname{tr}$ on $A$ such that, for $a \in A, \operatorname{tr}(a)$ corresponds to the trace per unit volume of $\pi_{\mathcal{T}}(a)$ (or of $\pi_{\mathcal{T}}^{S}(a)$ in a tight binding representation), then the integrated density of states at $E$ of the operator $\pi_{\mathcal{T}}(h)$ is given by $\operatorname{tr}\left(P_{-}(h)\right)$. The latter is the numerical gap-label of the gap $\Delta$ of $h$.

The traces on $A=C(\Xi) \rtimes_{\alpha} \mathbb{Z}^{d}$ are given by $\alpha$-invariant Borel-probability measures on $\Xi$. Indeed, if $\mu$ is such a measure then

$$
\operatorname{tr}_{\mu}\left(a_{n} u^{n}\right)=\int_{\Xi} a_{0}(\xi) d \mu(\xi)
$$

defines a trace on $A$ which is normalised, $\operatorname{tr}(1)=1$. If $\mu$ is ergodic, then for $\mu$-almost sure $\xi \in \Xi$ the trace per unit volume of $\pi_{\xi}(a)$ equals $\operatorname{tr}(a)$. In particular, if $\Xi$ carries a unique $\alpha$-invariant ergodic probability measure $\mu$ then for all $\xi$ the integrated density of $\pi_{\xi}(h)$ takes the same value $\operatorname{IDS}(E)=$ $\operatorname{tr}_{\mu}\left(P_{-}(h-E)\right)[8,25]$.

\subsubsection{Derivations and the winding number}

A *-derivation on $A$ is a densely defined linear map $d: A \rightarrow A$ which satisfies the Leibniz rule, $d(a b)=(d a) b+a(d b)$ and commutes with the $*$-operation. 
This implies that $d 1=0$ and thus for any invertible $Q$ (in the domain of $d$ ) $d Q^{-1}=-Q^{-1} d Q Q^{-1}$. Moreover, if $Q$ is unitary then $\left(Q^{-1} d Q\right)^{*}=-Q^{-1} d Q$.

If we have in addition a trace tr which is invariant under the derivation $d$, that is, $\operatorname{tr} \circ d=0$, then

$$
\mathcal{W}_{\mathrm{tr}, d}(Q):=\frac{1}{2 \pi i} \operatorname{tr}\left(Q^{-1} d Q\right)
$$

defines a real number which is stable under homotopy in $G L(A)$. It is called the winding number w.r.t. to $(\operatorname{tr}, d)$. $\mathcal{W}$ extends to a functional on $K_{1}(A)$, again by extending tr with the matrix trace if $Q \in G L\left(M_{n}(A)\right)$ [11].

There is a little subtlety related to the fact that $d$ is only densely defined. We need to be sure that the homotopy classes of invertibles in the domain of $d$ are in bijection to the homotopy classes of $G L(A)$. This can be done in the above cases [11].

\subsection{Bulk boundary correspondence}

A tiling covers the whole space $\mathbb{R}^{d}$ and so models a material which does not have a boundary. A real material has, of course, a boundary. The pattern equivariant operators above describe therefore properties in the interior of the material, that is, properties which do not depend on its boundary. Such properties are referred to as bulk properties. The bulk-boundary correspondence principle states that certain bulk properties correspond to boundary properties, i.e. properties which are confined to the boundary of the material.

The bulk-boundary correspondence, in the form we discuss it, is of topological nature. It is a correspondence between the topological invariants of the material when seen without boundary and topological invariants localised near the boundary. It manifests itself through a so-called boundary map in algebraic topology which maps the elements of the $K$-group of our algebra into the $K$-group of an algebra which contains the operators describing the boundary physics.

The topological nature of the bulk-boundary correspondence has physical consequences. Since topological invariants are robust against perturbations it explains the emergence of robust boundary spectrum in topological insulators.

There is a continuous and a discrete version of the bulk boundary correspondence. We discuss the discrete version for Wang tilings which is based on the Toeplitz extension. 


\subsubsection{General philosophy}

The bulk-boundary correspondence was first formulated by Hatsugai for the Quantum Hall Effect [20] in the context of band theory for periodic tight binding operators. Its topological nature and universality comes to full light through its formulation based on the $K$-theory of $C^{*}$-algebras. This was first formulated for the Quantum Hall Effect in [31], and later put into a general framework [30]. Today it is quite developped and we can refer the reader to the book [36] for further information.

Let $A$ be the algebra containing all pattern equivariant operators of the tiling describing the spatial structure of the material. The bulk invariants, that is the topological invariants associated to pattern equivariant operators, are elements of $K_{*}(A)$. The first goal is to find a short exact sequence

$$
0 \rightarrow J \rightarrow E \stackrel{q}{\rightarrow} A \rightarrow 0
$$

such that the elements of the ideal $J$ can be represented as operators localised at the boundary. The boundary map $\delta$ from the 6 -term exact sequence associated with the above short exact sequence

$$
\delta_{i}: K_{i}(A) \rightarrow K_{i+1}(J)
$$

maps the bulk invariants to invariants which are expressed in terms of operators of $J$ and therefore localised at the boundary. This is the heart (abstract form) of the bulk boundary correspondence. While there is no reason for $\delta_{i}$ to define a one-to-one correspondence, it will define a correspondence between some of the invariants.

Let us have a closer look at the boundary maps $\delta_{i}$. Let $h \in G L(A)^{s . a .}$, the Hamiltonian of an insulator. It has a gap $\Delta$ at 0 and the inverse of the gap width $|\Delta|$ is a characteristic time $t_{\Delta}=\frac{2 \pi}{|\Delta|}$. $h$ defines an element of $K_{0}(A)$ or, if it has chiral symmetry, an element of $K_{1}(A)$. To construct the image of these elements under the boundary map we need a lift $\hat{h} \in E^{s . a}$ of $h$, that is, $q(\hat{h})=h$.

We consider first the situation $\delta_{0}=\exp$, the so-called exponential map. It is given by

$$
\exp ([h])=\left[U_{\Delta}\right], \quad U_{\Delta}=P_{\Delta}(\hat{h})^{\perp}-e^{-i t_{\Delta}\left(\hat{h}-E_{0}\right)} P_{\Delta}(\hat{h})
$$

where $E_{0}=\inf \Delta$ and $P_{\Delta}(\hat{h})$ is the spectral projection of $\hat{h}$ to energies in $\Delta$ and $P_{\Delta}(\hat{h})^{\perp}$ its orthocomplement. The homotopy class of $U_{\Delta}$ does not depend on the chosen lift $\hat{h}$. By construction $U_{\Delta}-1$ belongs to the ideal $J$. If $\hat{h}$ is invertible then $[\hat{h}] \in K_{0}(E)$ is a preimage of $[h]$ and $\exp ([h])$ is trivial. If however no invertible lift can be found, then $\hat{h}$ has spectrum inside the gap $\Delta$ of $h$, and $U_{\Delta}$ is the unitary of time evolution by the characteristic time $t_{\Delta}$ of the states of $\hat{h}$ in $\Delta$. More generally, if $\hat{h}$ has a spectral gap overlapping 
with $\Delta$ and it is not protected by chiral symmetry, then it is homotopic to an invertible lift and so we see that non-triviality of $\exp ([h])$ implies that any self-adjoint lift $h$ in $E$ must have spectrum covering $\Delta$. It then follows that the spectrum of $U_{\Delta}$ covers the circle of complex numbers of absolute value 1 and no homotopy can retract that spectrum to a point.

We consider the case $\delta_{1}=$ ind, referred to as the index map. As already mentionned $h \in G L(A)^{\text {s.a. }}$ defines an element of $K_{1}(A)$ if it has so-called chiral symmetry and we consider topological phases which are protected under this symmetry. We assume that the chiral symmetry is inner and thus given by a generator $\Gamma$, a self-adjoint unitary, and $h$ anti-commutes with $\Gamma$. It gives rise to an isomorphism between $A$ and $M_{2}\left(A_{++}\right)$where $A_{++}=\Pi_{+} A \Pi_{+}$, $\Pi_{ \pm}=\frac{1}{2}(1 \pm \Gamma)$. Written as an element of $M_{2}\left(A_{++}\right), h$ and $\Gamma$ have the form

$$
h=\left(\begin{array}{cc}
0 & Q^{*} \\
Q & 0
\end{array}\right), \quad \Gamma=\left(\begin{array}{cc}
1 & 0 \\
0 & -1
\end{array}\right)
$$

and $Q \in G L\left(A_{++}\right)$. The homotopy class of $Q$ defines an element of $K_{1}\left(A_{++}\right) \cong$ $K_{1}(A)$ ( $A$ and $A_{++}$are strong Morita equivalent). Now the index map is given as follows

$$
\operatorname{ind}[Q]=\left[P_{\Delta}(\hat{h})^{\perp} \Gamma-P_{\Delta}(\hat{h})\left(\cos \left(t_{\Delta} \hat{h}\right) \Gamma-\sin \left(t_{\Delta} \hat{h}\right)\right)\right]
$$

as one computes using van Daele's formula applied to the class of $h[13]^{4}$. If $\hat{h}$ is invertible and has chiral symmetry then $\operatorname{ind}([Q])$ is trivial. Conversely, non-triviality of ind $([Q])$ implies that any lift $\hat{h} \in E$ with chiral symmetry must have spectrum at energy 0 . Finally, if 0 is an isolated point of the spectrum of $\hat{h}$ then the spectral projection $P_{0}(\hat{h})$ of $\hat{h}$ onto energy 0 is an element of $J$ and $P_{\Delta}(\hat{h})^{\perp} \Gamma-P_{\Delta}(\hat{h})\left(\cos \left(t_{\Delta} \hat{h}\right) \Gamma-\sin \left(t_{\Delta} \hat{h}\right)\right)$ homotopic to $P_{0}(\hat{h})^{\perp}-P_{0}(\hat{h})$, indeed, a homotopy is given by contracting $t_{\Delta} \hat{h}$ to $P_{0}(\hat{h})+$ $\frac{\pi}{2}\left(P_{>0}(\hat{h})-P_{<0}(\hat{h})\right)$.

\subsubsection{The Toeplitz extension as half space algebra}

We now specify to the algebra $A=C(\Xi) \rtimes_{\alpha} \mathbb{Z}^{d}$ for a Wang tiling. We follow $[31,30,36]$ to describe an extension of this algebra which describes the physics on half-space, the proto type of space with a boundary. We choose one direction in $\mathbb{Z}^{d}$, let's say along the $d$ 'th coordinate, and define the boundary to be perpendicular to it. The material with boundary is now described by the restriction of the tiling to half space, that is, we restrict the symbolic content $\xi \in \Xi$ to $\mathbb{Z}^{d-1} \times \mathbb{N}$. We can rewrite $A=B \rtimes_{\beta} \mathbb{Z}$ where $B=C(\Xi) \rtimes_{\alpha} \| \mathbb{Z}^{d-1}$ and $\beta=\alpha^{\perp}$. We argue that the Toeplitz extension $\mathcal{T}(B, \beta)$ describes the physics

4 In [36] one finds a formula for the same class with a different representative, the above is also be valid if $A$ is a real $C^{*}$-algebra. 
on the half space. This is best seen by looking at its iterated induced representation. Recall that the physically motivated representation (1.6) is the iterated induced representation of $\rho_{T}: C(\Xi) \rightarrow \mathbb{C}, \rho_{T}(f) \psi(x)=f([T ; x]) \psi(x)$ on $\ell^{2}\left(\mathbb{Z}^{d}\right)$. Applying this idea to $\mathcal{T}(B, \beta)$ we iterate the induction on $\rho_{T} d-1$ of times to get a representation of $B=C(\Xi) \rtimes_{\alpha} \| \mathbb{Z}^{d-1}$ on $\ell^{2}\left(\mathbb{Z}^{d-1}\right)$ and then once for the Toeplitz extension to get a representation of $\mathcal{T}(B, \beta)$ on $\ell^{2}\left(\mathbb{Z}^{d-1} \times \mathbb{N}\right)$. The operators in this representation are precisely the pattern equivariant operators restricted to the half space. The elements of the ideal $J$ in the short exact sequence are restrictions of pattern equivariant operators to the half space which are non-zero only near the boundary.

The exact sequence in $K$-theory arising from the Toeplitz extension (1.8) gives rise to the bijective correspondences

$$
K_{i}\left(C(\Xi) \rtimes_{\alpha} \mathbb{Z}^{d}\right) / \imath_{*} K_{i}\left(C(\Xi) \rtimes_{\alpha \|} \mathbb{Z}^{d-1}\right) \stackrel{\delta_{i}}{\cong} I_{\alpha_{*}^{\perp}} K_{i-1}\left(C(\Xi) \rtimes_{\alpha \|} \mathbb{Z}^{d-1}\right) .
$$

This is the $K$-theoretic bulk boundary correspondence. We refer to the case with $i=0$ as even, and that with $i=1$ as the odd correspondence. Note that the boundary maps are explicit, as a lift $\hat{h}$ of $h$ in the Toeplitz extension is simply given by replacing the translation operator in the $d$ th coordinate by its truncation to $\mathbb{Z}^{d-1} \times \mathbb{N}$.

\subsubsection{One-dimensional examples}

If the dimension is $d=1$ then (1.11) becomes, for odd $i$,

$$
K_{1}\left(C(\Xi) \rtimes_{\alpha} \mathbb{Z}\right) / \imath_{*} K_{1}(C(\Xi)) \stackrel{\text { ind }}{\cong} I_{\alpha_{*}} K_{0}(C(\Xi)) .
$$

Since $\Xi$ is the closure of a single orbit, $I_{\alpha_{*}} K_{0}(C(\Xi))$ has a single generator, the class of -1 , which corresponds to a Hamiltonian whose spectrum is purely negative. Likewise the invariants of $K_{0}\left(\mathbb{C}^{k}\right)$ are generated by the class of -1 . One computes that $[u]$ is a pre-image for $[-1]$, that is, ind $([u])=[-1]$.

Let $\mu$ be an ergodic $\alpha$-invariant probability measure on $\Xi$ and recall that it defines a trace $\operatorname{tr}$ on $C(\Xi)$ and $C(\Xi) \rtimes_{\alpha} \mathbb{Z}$. Consider the *-derivation $\delta: C(\Xi)_{\alpha} \mathbb{Z} \rightarrow C(\Xi)_{\alpha} \mathbb{Z}$ given by $\delta\left(b u^{n}\right)=2 \pi i n b u^{n}$. The trace $\operatorname{tr}$ defines a tracial state on $K_{0}(C(\Xi))$ and, together with the derivation $\delta$ a winding number $\mathcal{W}_{\operatorname{tr}, \delta}$ on $K_{1}\left(C(\Xi) \rtimes_{\alpha} \mathbb{Z}\right)$. One finds that $\mathcal{W}_{\operatorname{tr}, \delta}(u)=\operatorname{tr}(1)$. Thus applying the winding number to the left hand side and the tracial state to the right hand side of (1.12) one obtains the odd numerical bulk-boundary correspondence

$$
\mathcal{W}_{\operatorname{tr}, \delta}([Q])=\operatorname{tr}(\operatorname{ind}[Q])
$$


This has the following physical interpretation: If $h=\left(\begin{array}{cc}0 & Q^{*} \\ Q & 0\end{array}\right)$ is the Hamiltonian with chiral symmetry and gap $\Delta$ at energy 0 then the spectrum of any lift $\hat{h}$ of $h$ has at most finitely many values in $\Delta$. Moreover, these values are eigenvalues to eigenfunctions localized at the edge. Hence ind $[Q]=\left[P_{0}(\hat{h})^{\perp}-P_{0}(\hat{h})\right]$ and thus $\operatorname{tr}(\operatorname{ind}[Q])$ is equal to the number of 0 energy eigenstates of $\hat{h}$. We thus have the following well-known result (see also $[35,36])$.

Odd bulk boundary correspondence

The winding number $\mathcal{W}_{\operatorname{tr}, \delta}$ of $Q$ is the number of 0 energy boundary states of $\hat{h}$.

For even $i(1.11)$ is

$$
K_{0}\left(C(\Xi) \rtimes_{\alpha} \mathbb{Z}\right) / \imath_{*} K_{0}(C(\Xi)) \stackrel{\exp }{\cong} I_{\alpha_{*}} K_{1}(C(\Xi))
$$

Other than in the case of odd $i$ the situation depends on the nature of the hull $\Xi$. Again, given an ergodic $\alpha$-invariant measure on $\Xi$ we obtain a trace tr and a tracial state, now for the left hand side. To obtain a winding number for the right hand side we need a derivation on $C(\Xi)$.

We discuss these issues here for the one-dimensional systems of Section 1.2.3.

1. We consider a periodic sequence $\xi$. If the period is $k$ the discrete hull $\Xi$ consists of $k$ isolated points on which $\mathbb{Z}$ acts by a cyclic permutation. Hence $C(\Xi)=\mathbb{C}^{k}$ and the unique invariant probability measure is the uniform probability measure on $k$ points. But we have $K_{1}\left(\mathbb{C}^{k}\right)=0$ and hence the even bulk-boundary correspondence is trivial.

2 . We consider an almost periodic sequence $\xi$. The discrete hull $\Xi$ is homeomorphic to the circle $S$ and $\alpha$ is rotation by the irrational number $\theta \in[0,1]$. Now $K_{1}(C(S)) \cong \mathbb{Z}$ with generator given by the class of the function $z(s)=e^{2 \pi i s}$. Hence also the coinvariants and the invariants of $K_{1}(C(S))$ are generated by $[z]$. Furthermore, $K_{0}(C(S)) \cong \mathbb{Z}$ with generator given by the class of -1 , which then is also the generator of the coinvariants and the invariants of $K_{0}(C(S)) . K_{1}\left(C(S) \rtimes_{\alpha} \mathbb{Z} \cong \mathbb{Z}^{2}\right.$ with generators $[z]$ and $[u] . K_{0}\left(C(S) \rtimes_{\alpha} \mathbb{Z} \cong \mathbb{Z}^{2}\right.$ with generators [-1] and $\left[h_{\theta}\right]$. The second generator is the class of the function $h_{\theta}=1-2 P_{\theta}$ where $P_{\theta}$ is the famous Rieffel projection [38].

The exponential map can be computed on the generators and one finds that the even bulk-boundary correspondence is given by

$$
\exp \left(\left[h_{\theta}\right]\right)=-[z], \quad \exp ([-1])=0 .
$$

Since $\theta$ is irrational, the unique ergodic invariant measure on the circle is the Lebesgues measure. Furthermore, functions of class $C^{1}$ in $C(S)$ can 
be derived and thus we have a densely defined derivation $\partial_{s}$. Applying the resulting tracial state to the left and the winding number $\mathcal{W}_{\operatorname{tr}, \partial_{\mathrm{s}}}$ to the right hand side we get $\operatorname{tr}(1)=1, \operatorname{tr}\left(P_{\theta}\right)=\theta[38], \mathcal{W}_{\operatorname{tr}, \partial_{s}}(u) \stackrel{,_{s}}{=} 0$, $\mathcal{W}_{\operatorname{tr}, \partial_{s}}(z)=1$, so that the even numerical bulk boundary correspondence is given by

$$
\operatorname{tr}([h])+\theta \mathcal{W}_{\operatorname{tr}, \partial_{s}}(\exp [h]) \in \mathbb{N} .
$$

This has the following interpretation. The bulk invariant $\operatorname{tr}([h])$ is the IDS of $h$ at energy 0 (Fermi energy) and it can take real values of the form $m \theta+N$ where $N, m \in \mathbb{N}$ and $N$ is such that $m \theta+N \in[0,1]$. Thus, except if $m=0, N$ is determined by the value of $m$ as $\theta$ is irrational. The boundary invariant $\mathcal{W}_{\operatorname{tr}, \partial_{s}}(\exp [h])$ is a response coefficient related to a mechanical force. The derivation $\partial_{s}$ is the infinitesimal generator of translation in the hull and translation has the effect of changing the position of the atoms (boundary points of the tiles) in the structure. This atomic motion corresponds to a variation of the potential which the electrons at the edge are subjected to, and hence they feel a gradient force. The above winding number of $U_{\Delta}$ corresponds to the expectation value of the gradient force on all edge states with energy inside $\Delta$, integrated over one cycle of the circle $\Xi$, in units of $|\Delta|[28]$. We summarize:

\section{Even bulk boundary correspondence}

If $m \theta+N$ is the IDS of $h$ at 0 then the work exhibited by the atomic motion through a cycle around $S$, on the edge states of energy inside $\Delta$,

$$
\text { is }-m|\Delta| \text {. }
$$

3. We consider a quasi periodic sequence $\xi$, more precisely a Sturmian sequence. Now $\Xi$ is the cut up circle $S_{c}$. It is totally disconnected and therefore $K_{0}(C(\Xi)) \cong \mathcal{C}_{\alpha} C(\Xi, \mathbb{Z})$ and $K_{1}(C(\Xi)) \cong 0$. It follows that the even bulk-boundary correspondence is trivial, as in the periodic case. There is nevertheless a difference with the periodic case, as namely $C_{\alpha} C(\Xi, \mathbb{Z})$ has two generators. Next to the class [-1] it contains the class $\left[1-2 \chi_{\theta}\right]$ where $\chi_{\theta}$ is the characteristic function on a clopen subset of $\Xi$ which has measure $\theta$ w.r.t. the unique ergodic invariant probability measure.

4. The reason why the even $\mathrm{BBC}$ is trivial in the periodic and the quasiperiodic case is, that in these cases the $K_{1}$-group of the ideal in the Toeplitz extension is trivial. This is due to the fact that the connected component of a point in the hull contains only that point. There is also no obvious definition of a winding number, as there is no notion of continuous translation in such spaces, any translation pushes a point out of its connected component. Likewise we can't define a gradient force. It is here where the augmented system comes into play.

Recall that we can make the phason flips continuous by augmenting the system, that is, adding sequences in which the phason flip is only fractionally carried out. Since $\tilde{\Xi}$ is a circle, $K_{1}(C(\tilde{\Xi}))$ is non-trivial and the 
even bulk boundary correspondence becomes non-trivial as well. But this means that we abandon the simple Toeplitz extension for $C(\Xi) \rtimes_{\alpha} \mathbb{Z}$ and replace it by a more complicated SES. We outline the construction refering the reader to [28] for more details.

The augmented hull $\tilde{\Xi}$ contains $\Xi$ as a closed shift-invariant subset. The Toeplitz extension $\mathcal{T}(C(\tilde{\Xi}), \alpha)$ of the augmented model is therefore also an extension of $C(\Xi) \rtimes_{\alpha} \mathbb{Z}$. If we take this extension for (1.10) then we end up with the SES

$$
0 \rightarrow C_{\alpha_{*}} K_{0}(C(\tilde{\Xi})) \stackrel{\tilde{i}_{*}}{\longrightarrow} K_{0}\left(C(\Xi) \rtimes_{\alpha} \mathbb{Z}\right) \stackrel{\text { exp }}{\longrightarrow} I_{\alpha_{*}} K_{1}(C(\tilde{\Xi})) \rightarrow 0
$$

where $\tilde{i}_{*}$ is induced by the inclusion after restriction $C(\tilde{\Xi}) \rightarrow C(\Xi) \rightarrow$ $C(\Xi) \rtimes_{\alpha} \mathbb{Z}$. Again $\alpha_{*}$ is the identity and $K_{0}(C(\tilde{\Xi})) \cong K_{1}(C(\tilde{\Xi})) \cong \mathbb{Z}$. Of the two generators of $K_{0}\left(C(\Xi) \rtimes_{\alpha} \mathbb{Z}\right)$, the class [-1] and the class $\left[1-2 \chi_{\theta}\right]$, the first lies in the kernel of the exponential map and for the second one finds $\exp \left(\left[1-2 \chi_{\theta}\right]\right)=-[\tilde{z}]$ where $\tilde{z}: \tilde{\Xi} \rightarrow \mathbb{C}$ is the function with winding number -1 .

There is a unique ergodic invariant probability measure on $\Xi$ (when identifying $\Xi$ with $S_{c}$, which is from the point of view of measure theory the same as $S$, the above measure is the Lebesgues measure) which we use to define the trace and tracial state on $K_{0}\left(C(\Xi) \rtimes_{\alpha} \mathbb{Z}\right.$. To define the winding number on $C(\tilde{\Xi})$ we use the derivation w.r.t. the parameter $t$ describing

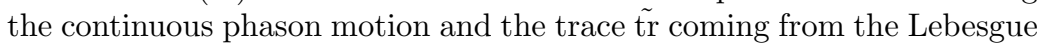
measure on the added intervals (see [28] for more details). We then get the non-trivial even bulk boundary correspondence $\operatorname{tr}\left(\left[\chi_{\theta}\right]\right)=-\theta \mathcal{W}_{\tilde{\mathrm{tr}}, \partial_{t}}([\tilde{z}])$ modulo $\mathbb{Z}$. As in the almost periodic case the winding number of the boundary invariant has a physical interpretation; it is the work the phason flips exhibit on the boundary states with energy in the gap $\Delta$, in units of $|\Delta|$.

\section{Even bulk boundary correspondence [28]}

$$
\begin{gathered}
\text { If } n+m \theta \text { is the IDS of } h \text { at } 0 \text { then the work exhibited by a cycle of } \\
\text { phason flips on the edge states of energy inside } \Delta \text { is }-m|\Delta| \text {. }
\end{gathered}
$$

This identity has been experimentally observed [2].

We summarize the $K$-theoretical results in the following table. Here $A=$ $C(\theta) \rtimes_{\alpha} \mathbb{Z}$ is the discrete tiling algebra and $J$ the ideal in the SES. In the first three cases, $J=\mathcal{K} \otimes C(\Xi)$ the ideal in the Toeplitz extension $\mathcal{T}(C(\Xi), \alpha)$ whereas in the fourth case $J$ is the kernel of $\mathcal{T}(C(\tilde{\Xi}), \alpha) \rightarrow C(\Xi) \rtimes_{\alpha} \mathbb{Z}$. The table shows the generators as denoted in the text above. 


\begin{tabular}{|l||c|c|c||c|c|c|}
\hline case & $C_{\alpha_{*}} K_{0}(J)$ & $K_{0}(A)$ & $I_{\alpha_{*}} K_{1}(J)$ & $C_{\alpha_{*}} K_{1}(J)$ & $K_{1}(A)$ & $I_{\alpha_{*}} K_{0}(J)$ \\
\hline \hline$k$-periodic & {$[-1]$} & {$[-1]$} & 0 & 0 & {$[u]$} & {$[-1]$} \\
\hline almost-per. & {$[-1]$} & {$[-1],\left[h_{\theta}\right]$} & {$[z]$} & {$[z]$} & {$[z],[u]$} & {$[-1]$} \\
\hline quasi-per. & {$[-1],\left[1-2 \chi_{\theta}\right]$} & {$[-1],\left[1-2 \chi_{\theta}\right]$} & 0 & 0 & {$[u]$} & {$[-1]$} \\
\hline augmented & {$[-1]$} & {$[-1],\left[1-2 \chi_{\theta}\right]$} & {$[\tilde{z}]$} & {$[\tilde{z}]$} & {$[\tilde{z}],[u]$} & {$[-1]$} \\
\hline
\end{tabular}

\section{References}

1. Anderson, J. E., Putnam, I. F. (1998). Topological invariants for substitution tilings and their associated $C^{*}$-algebras. Ergodic Theory and Dynamical Systems, 18(3), 509-537.

2. F. Baboux, E. Levy, A. Lemaitre, C. Gómez, E. Galopin, L. L. Gratiet, I. Sagnes, A Amo, J. Bloch, E. Akkermans, Measuring topological invariants from generalized edge states in polaritonic quasicrystals, Phys. Rev. B 95, 161114(R) (2017).

3. Avila, A., Jitomirskaya, S. (2009). The ten Martini problem. Annals of Mathematics, 303-342.

4. Baake, M., Grimm, U. (2013). Aperiodic Order (Vol. 1). Cambridge University Press.

5. Baake, M., Schlottmann, M., Jarvis, P. D. (1991). Quasiperiodic tilings with tenfold symmetry and equivalence with respect to local derivability. Journal of Physics A: Mathematical and General, 24(19), 4637.

6. J. Bellissard, K-theory of $C^{*}$-Algebras in solid state physics, Statistical mechanics and field theory: mathematical aspects, (Springer, Berlin, Heidelberg, 1986 , 99-156).

7. J. Bellissard, Gap labeling theorems for Schr'odinger operators, in M. Waldschmidt, P. Moussa, J.-M. Luck, C. Itzykson eds., "From number theory to physics," (Springer, Berlin, 1995).

8. Bellissard, J., Herrmann, D. J. L., Zarrouati, M. (2000). Hull of aperiodic solids and gap labelling theorems. Directions in mathematical quasicrystals, 13, 207258.

9. B. Blackadar. K-theory for operator algebras. Second edition. Mathematical Sciences Research Institute Publications, 5. Cambridge University Press, Cambridge, 1998.

10. Clark, A., Sadun, L. (2006). When shape matters: deformations of tiling spaces. Ergodic Theory and Dynamical Systems, 26(1), 69-86.

11. A. Connes, Non-Commutative Geometry, (Acad. Press, San Diego, 1994).

12. A. van Daele, K-theory for graded Banach algebras I. The Quarterly Journal of Mathematics 39.2 (1988): 185-199.

13. A. van Daele, K-theory for graded Banach algebras II. Pacific J. Math. 1352 (1988) 377-392

14. Damanik, D., Embree, M., Gorodetski, A. (2015). Spectral properties of Schrödinger operators arising in the study of quasicrystals. In Mathematics of Aperiodic Order (pp. 307-370). Birkhäuser, Basel.

15. Davidson, K. (1996) $\mathrm{C}^{*}$-algebras by example. Vol. 6. American Mathematical Soc..

16. M. R. Herman, Sur la conjugaison différentiable des difféomorphismes du cercle à des rotations, Pub. Math. IHES 49 5-234 (1979).

17. Forrest, A., Hunton, J., Kellendonk, J. (2002). Topological invariants for projection method patterns (No. 758). American Mathematical Soc.. 
18. Computer code written by Franz Gähler, Universität Bielefeld.

19. Gähler, F., Hunton, J., Kellendonk, J. (2013). Integral cohomology of rational projection method patterns. Algebraic \& Geometric Topology, 13(3), 1661-1708.

20. Hatsugai, Y. (1993) Chern number and edge states in the integer quantum Hall effect. Physical Review Letters 71 (22), 3697.

21. Higson, N., Roe, J. (2000). Analytic K-homology. OUP Oxford.

22. Hunton, J. (2015). Spaces of projection method patterns and their cohomology. In Mathematics of aperiodic order (pp. 105-135). Birkhäuser, Basel.

23. S. Jitomirskaya. Metal-insulator transition for the almost Mathieu operator. Ann. of Math. 150 (1999), 11591175.

24. Kalugin, P. (2005). Cohomology of quasiperiodic patterns and matching rules. Journal of Physics A: Mathematical and General, 38(14), 3115.

25. Kellendonk, J. (1995). Noncommutative geometry of tilings and gap labelling. Reviews in Mathematical Physics, 7(07), 1133-1180.

26. Kellendonk, J. (2003). Pattern-equivariant functions and cohomology. Journal of Physics A: Mathematical and General, 36(21), 5765.

27. Kellendonk, J. (2008). Pattern equivariant functions, deformations and equivalence of tiling spaces. Ergodic Theory and Dynamical Systems, 28(4), 1153-1176.

28. Kellendonk, J., Prodan, E. (2019). Bulk-boundary correspondence for Sturmian Kohmoto like models. Annales Henri Poincar DOI 10.1007/s00023-019-00792-5

29. Kellendonk, J., Putnam, I. F. (1999). Tilings, $C^{*}$-algebras and K-theory. In: Directions in Mathematical Quasicrystals, Baake, M., Moody, R.V. (eds.), CRM Monograph Series, Volume 13, Providence, RI: Amer. Math. Soc., 2000, pp. 177206

30. J. Kellendonk, S. Richard, Topological boundary maps in physics, in Perspectives in operator algebras and mathematical physics, 105-121, Theta Ser. Adv. Math. 8, (Theta, Bucharest, 2008).

31. J. Kellendonk, T. Richter, H. Schulz-Baldes, Edge current channels and Chern numbers in the integer quantum Hall effect, Rev. Math. Phys. 14, 87-119 (2002).

32. J. Kellendonk, On the $C^{*}$-Algebraic Approach to Topological Phases for Insulators. Annales Henri Poincar. Vol. 18. No. 7 (2017), 2251-2300.

33. Last, Y. (2005). Spectral theory of Sturm-Liouville operators on infinite intervals: a review of recent developments. In Sturm-Liouville Theory (pp. 99-120). Birkhäuser Basel.

34. M. Pimsner, D. Voiculescu. Exact sequences for K-groups of certain cross products of $C^{*}$-algebras, J. Op. Theory 4 93-118 (1980).

35. Prodan, E., Schulz-Baldes, H. (2016). Non-commutative odd Chern numbers and topological phases of disordered chiral systems. Journal of Functional Analysis, 271(5), 1150-1176.

36. Prodan, E., Schulz-Baldes, H. (2016). Bulk and boundary invariants for complex topological insulators. Springer.

37. Renault, J. (2006). A groupoid approach to $\mathrm{C}^{*}$-algebras (Vol. 793). Springer.

38. Rieffel, M. (1981). $C^{*}$-algebras associated with irrational rotations. Pacific Journal of Mathematics, 93(2), 415-429.

39. M Rordam, F Larsen, N.J. Laustsen An Introduction to K-theory of $C^{*}$-algebras, Cambridge University Press (2000).

40. Sadun, L. A. (2008). Topology of tiling spaces (Vol. 46). American Mathematical Soc..

41. Sadun, L. (2015). Cohomology of Hierarchical Tilings. In Mathematics of Aperiodic Order (pp. 73-104). Birkhäuser, Basel.

42. Sadun, L., Williams, R. F. (2003). Tiling spaces are Cantor set fiber bundles. Ergodic Theory and Dynamical Systems, 23(1), 307-316. 